\title{
THE EIGHTH DATA RELEASE OF THE SLOAN DIGITAL SKY SURVEY: FIRST DATA FROM SDSS-III
}

Hiroaki Aihara ${ }^{1}$, Carlos Allende Prieto $^{2,3}$, Deokkeun An ${ }^{4}$, Scott F. Anderson $^{5}$, Éric Aubourg ${ }^{6,7}$, Eduardo Balbinot ${ }^{8,9}$, Timothy C. Beers ${ }^{10}$, Andreas A. Berlind ${ }^{11}$, Steven J. Bickerton ${ }^{12}$, Dmitry Bizyaev ${ }^{13}$,

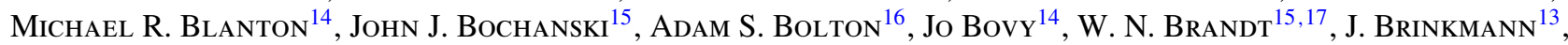
Peter J. Brown ${ }^{16}$, Joel R. Brownstein ${ }^{16}$, Nicolas G. Busca ${ }^{6}$, Heather Campbell ${ }^{18}$, Michael A. Carr ${ }^{12}$, Yanmei Chen ${ }^{19}$, Cristina Chiappini ${ }^{9}, 20,21$, Johan Comparat ${ }^{22}$, Natalia Connolly ${ }^{23}$, Marina Cortes ${ }^{24}$, Rupert A. C. Croft $^{25}$, Antonio J. Cuesta ${ }^{26}$, Luiz N. da Costa ${ }^{9,27}$, James R. A. Davenport ${ }^{5}$, Kyle Dawson $^{16}$, Saurav Dhital ${ }^{11}$, Anne Ealet $^{28}$, Garrett L. Ebelke ${ }^{13,29}$, Edward M. Edmondson ${ }^{18}$, Daniel J. Eisenstein ${ }^{30,31}$, STEPhanie Escoffier $^{28}$,

Massimiliano Esposito ${ }^{2,3}$, Michael L. Evans ${ }^{5}$, Xiaohui Fan $^{30}$, Bruno Femenía Castella B $^{2,3}$, Andreu Font-Ribera ${ }^{32}$,

Peter M. Frinchaboy ${ }^{33}$, Jian Ge ${ }^{34}$, Bruce A. Gillespie ${ }^{13}$, G. Gilmore ${ }^{35}$, Jonay I. GonZález HernándeZ ${ }^{2}$, J. Richard Gott ${ }^{12}$, Andrew Gould ${ }^{36}$, Eva K. Grebel ${ }^{37}$, James E. GunN ${ }^{12}$, Jean-Christophe Hamilton $^{6}$, Paul Harding ${ }^{38}$, David W. Harris ${ }^{16}$, Suzanne L. Hawley ${ }^{5}$, Frederick R. Hearty ${ }^{39}$, Shirley Ho ${ }^{24}$, David W. Hogg ${ }^{14}$, Jon A. Holtzman ${ }^{29}$,

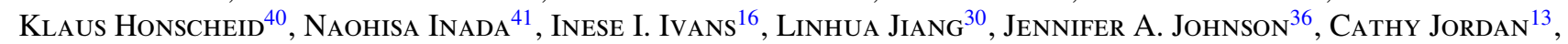
Wendell P. Jordan ${ }^{13,29}$, Eyal A. Kazin ${ }^{14}$, David KirkBY ${ }^{42}$, Mark A. Klaene ${ }^{13}$, G. R. KNapP ${ }^{12}$, Jean-Paul Kneib ${ }^{22}$, C. S. KochaneK ${ }^{36}$, Lars Koesterke ${ }^{43}$, Juna A. Kollmeier ${ }^{44}$, Richard G. Kron ${ }^{45}, 46$, Hubert Lampeitl ${ }^{18}$, Dustin Lang ${ }^{12}$, Jean-Marc Le GofF ${ }^{7}$, Young Sun LeE ${ }^{10}$, Yen-Ting Lin ${ }^{1,47}$, Daniel C. Long ${ }^{13}$, Craig P. Loomis ${ }^{12}$, Sara Lucatello ${ }^{48}$,

Britt LundGren $^{26}$, Robert H. Lupton ${ }^{12}$, Zhibo MA ${ }^{38}$, Nicholas MacDonald ${ }^{5}$, Suvrath Mahadevan ${ }^{15,49}$, Marcio A. G. Maia ${ }^{9,27}$, Martin MaKler ${ }^{9,50}$, Elena Malanushenko ${ }^{13}$, Viktor Malanushenko ${ }^{13}$, Rachel Mandelbaum $^{12}$, Claudia Maraston ${ }^{18}$, Daniel Margala ${ }^{42}$, Karen L. Masters ${ }^{18}$, Cameron K. McBride $^{11}$, Peregrine M. McGehee ${ }^{51}$, Ian D. McGreer ${ }^{30}$, Brice Ménard ${ }^{1,52,53}$, Jordi Miralda-Escudé ${ }^{54,55}$, Heather L. Morrison ${ }^{38}$, F. Mullally ${ }^{12,56}$, Demitri Muna ${ }^{14}$, Jeffrey A. Munn ${ }^{57}$, Hitoshi Murayama ${ }^{1}$, Adam D. Myers ${ }^{58}$, Tracy Naugle $^{13}$, Angelo Fausti Neto ${ }^{8,9}$, Duy Cuong Nguren ${ }^{34}$, Robert C. Nichol ${ }^{18}$, Robert W. O'Connell ${ }^{39}$, Ricardo L. C. Ogando ${ }^{9}, 27$, Matthew D. Olmstead $^{16}$, Daniel J. Oravetz ${ }^{13}$, Nikhil Padmanabhan ${ }^{26}$, Nathalie Palanque-Delabrouille ${ }^{7}$, Kaike Pan ${ }^{13}$, Parul Pandey ${ }^{16}$, Isabelle PÂris ${ }^{59}$, Will J. Percival ${ }^{18}$, Patrick Petitjean ${ }^{59}$, Robert Pfaffenberger ${ }^{29}$, Janine Pforr ${ }^{18}$, Stefanie Phleps $^{60}$, Christophe Pichon ${ }^{59}$, Matthew M. Pieri ${ }^{36,61}$, Francisco Prada ${ }^{62}$, Adrian M. Price-Whelan ${ }^{14}$, M. Jordan Raddick ${ }^{53}$, Beatriz H. F. Ramos ${ }^{9,27}$, Céline Reylé ${ }^{63}$, James Rich ${ }^{7}$, Gordon T. Richards ${ }^{64}$, Hans-Walter Rix ${ }^{65}$, AnNie C. Robin ${ }^{63}$, Helio J. Rocha-Pinto ${ }^{9,66}$, Constance M. Rockosi ${ }^{67}$, Natalie A. RoE ${ }^{24}$, Emmanuel Rollinde ${ }^{59}$, Ashley J. Ross ${ }^{18}$,

Nicholas P. Ross ${ }^{24}$, Bruno M. Rossetto ${ }^{9,66}$, Ariel G. Sánchez ${ }^{60}$, Conor Sayres ${ }^{5}$, David J. Schlegel ${ }^{24}$,

Katharine J. Schlesinger ${ }^{36}$, SARah J. Schmidt ${ }^{5}$, Donald P. Schneider ${ }^{15,49}$, Erin Sheldon ${ }^{68}$, Yiping Shu ${ }^{16}$,

Jennifer Simmerer ${ }^{16}$, Audrey E. Simmons ${ }^{13}$, Thirupathi Sivarani ${ }^{34,69}$, STEPhanie A. Snedden $^{13}$, Jennifer S. Sobeck ${ }^{46}$, Matthias Steinmetz ${ }^{20}$, Michael A. Strauss ${ }^{12,72}$, Alexander S. Szalay ${ }^{53}$, Masayuki Tanaka $^{1}$, Aniruddha R. Thakar ${ }^{53}$, Daniel Thomas $^{18}$, Jeremy L. Tinker ${ }^{14}$, Benjamin M. Tofflemire ${ }^{5}$, Rita Tojeiro ${ }^{18}$, Christy A. Tremonti ${ }^{19}$, Jan VandenberG ${ }^{53}$, M. Vargas Magaña ${ }^{6}$, Licia Verde ${ }^{54,55}$, Nicole P. VogT ${ }^{29}$, David A. Wake ${ }^{26}$, Ji WanG $^{34}$, Benjamin A. Weaver ${ }^{14}$, David H. Weinberg ${ }^{36}$, Martin White ${ }^{70}$, Simon D. M. White ${ }^{71}$, Brian Yanny ${ }^{45}$, NAOKI YASUdA ${ }^{1}$, Christophe YeChE ${ }^{7}$, AND IDIT Zehavi ${ }^{38}$

${ }^{1}$ Institute for the Physics and Mathematics of the Universe, The University of Tokyo, 5-1-5 Kashiwanoha, Kashiwa, 277-8583, Japan

${ }_{2}^{2}$ Instituto de Astrofísica de Canarias, E38205 La Laguna, Tenerife, Spain

${ }^{3}$ Departamento de Astrofísica, Universidad de La Laguna, 38206, La Laguna, Tenerife, Spain

${ }^{4}$ Department of Science Education, Ewha Womans University, Seoul 120-750, Republic of Korea

${ }^{5}$ Department of Astronomy, University of Washington, Box 351580, Seattle, WA 98195, USA

${ }^{6}$ Astroparticule et Cosmologie (APC), Université Paris-Diderot, 10 rue Alice Domon et Léonie Duquet, 75205 Paris Cedex 13, France ${ }^{7}$ CEA, Centre de Saclay, Irfu/SPP, F-91191 Gif-sur-Yvette, France

${ }^{8}$ Instituto de Física, UFRGS, Caixa Postal 15051, Porto Alegre, RS 91501-970, Brazil

${ }^{9}$ Laboratório Interinstitucional de e-Astronomia (LIneA), Rua Gal. José Cristino 77, Rio de Janeiro, RJ 20921-400, Brazil

${ }^{10}$ Department of Physics \& Astronomy and JINA: Joint Institute for Nuclear Astrophysics, Michigan State University, E. Lansing, MI 48824, USA

${ }^{11}$ Department of Physics and Astronomy, Vanderbilt University, Nashville, TN 37235, USA

${ }^{12}$ Department of Astrophysical Sciences, Princeton University, Princeton, NJ 08544, USA ${ }^{13}$ Apache Point Observatory, P.O. Box 59, Sunspot, NM 88349, USA

${ }^{14}$ Center for Cosmology and Particle Physics, New York University, 4 Washington Place, New York, NY 10003, USA

${ }^{15}$ Department of Astronomy and Astrophysics, 525 Davey Laboratory, The Pennsylvania State University, University Park, PA 16802, USA

${ }^{16}$ Department of Physics and Astronomy, University of Utah, Salt Lake City, UT 84112, USA

${ }^{17}$ Institute for Gravitation and the Cosmos, The Pennsylvania State University, University Park, PA 16802, USA

${ }^{18}$ Institute of Cosmology and Gravitation (ICG), Dennis Sciama Building, Burnaby Road, University of Portsmouth, Portsmouth, PO1 3FX, UK

${ }^{19}$ Department of Astronomy, University of Wisconsin-Madison, 475N. Charter St., Madison, WI 53703, USA

${ }^{20}$ Astrophysical Institute Potsdam, An der Sternwarte 16, 14482 Potsdam, Germany

${ }^{21}$ Istituto Nazionale di Astrofisica, Via G. B. Tiepolo 11, 34143 Trieste, Italy

${ }^{22}$ Laboratoire d'Astrophysique de Marseille, CNRS-Université de Provence, 38 rue F. Joliot-Curie, 13388 Marseille Cedex 13, France

${ }^{23}$ Department of Physics, Hamilton College, Clinton, NY 13323, USA

${ }^{24}$ Lawrence Berkeley National Laboratory, One Cyclotron Road, Berkeley, CA 94720, USA

${ }^{25}$ Bruce and Astrid McWilliams Center for Cosmology, Carnegie Mellon University, Pittsburgh, P.A. 15213, USA

${ }^{26}$ Yale Center for Astronomy and Astrophysics, Yale University, New Haven, CT 06520, USA

${ }^{27}$ Observatório Nacional, Rua Gal. José Cristino 77, Rio de Janeiro, RJ 20921-400, Brazil 


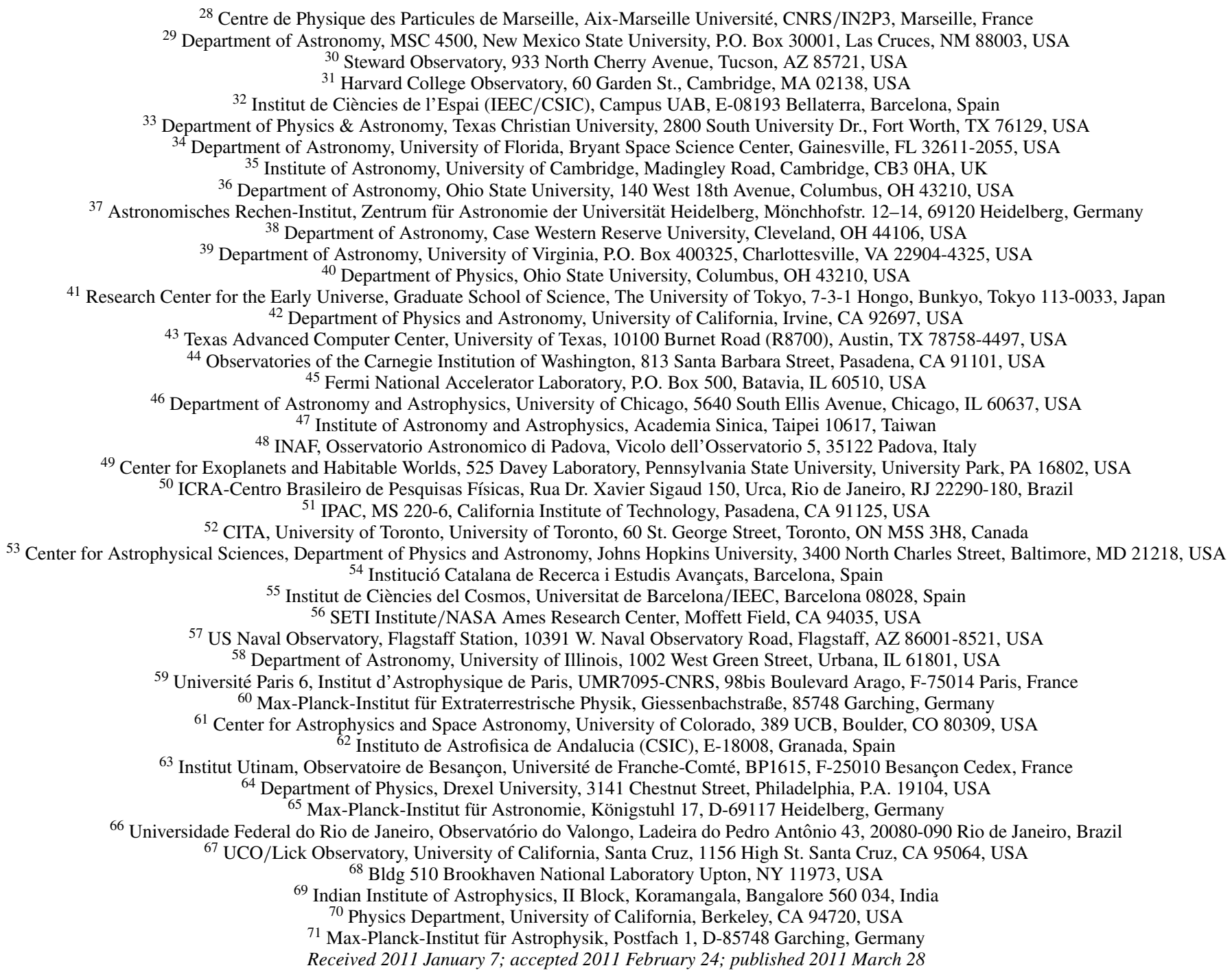

\begin{abstract}
The Sloan Digital Sky Survey (SDSS) started a new phase in 2008 August, with new instrumentation and new surveys focused on Galactic structure and chemical evolution, measurements of the baryon oscillation feature in the clustering of galaxies and the quasar Ly $\alpha$ forest, and a radial velocity search for planets around $\sim 8000$ stars. This paper describes the first data release of SDSS-III (and the eighth counting from the beginning of the SDSS). The release includes five-band imaging of roughly $5200 \mathrm{deg}^{2}$ in the southern Galactic cap, bringing the total footprint of the SDSS imaging to $14,555 \mathrm{deg}^{2}$, or over a third of the Celestial Sphere. All the imaging data have been reprocessed with an improved sky-subtraction algorithm and a final, self-consistent photometric recalibration and flat-field determination. This release also includes all data from the second phase of the Sloan Extension for Galactic Understanding and Exploration (SEGUE-2), consisting of spectroscopy of approximately 118,000 stars at both high and low Galactic latitudes. All the more than half a million stellar spectra obtained with the SDSS spectrograph have been reprocessed through an improved stellar parameter pipeline, which has better determination of metallicity for high-metallicity stars.
\end{abstract}

Key words: atlases - catalogs - surveys

\section{INTRODUCTION}

The Sloan Digital Sky Survey (SDSS; York et al. 2000) saw first light in 1998 May and has been in routine survey operation mode since 2000 April. It uses a $2.5 \mathrm{~m}$ telescope with an unvignetted $3^{\circ}$ field of view (Gunn et al. 2006) at Apache

\footnotetext{
${ }^{72}$ Corresponding author.
}

Point Observatory (APO) in Southern New Mexico, which is dedicated to wide-angle surveys of the sky. The first and second phases of the survey (SDSS-I and SDSS-II) were carried out with two instruments: a drift-scan imaging camera (Gunn et al. 1998 ) with 30 CCDs imaging in five filters (ugriz; Fukugita et al. 1996) and a pair of double spectrographs, fed by 640 optical fibers. The imaging data, essentially all of which have been taken under photometric and good-seeing conditions (Ivezić 
et al. 2004; Padmanabhan et al. 2008; see also Hogg et al. 2001), now cover more than $14,500 \mathrm{deg}^{2}$ in five filters (of which about $11,600 \mathrm{deg}^{2}$ were observed as part of SDSS-I/II), or roughly one-third of the Celestial Sphere. The $50 \%$ completeness limit for point sources is $r=22.5$. The data have been analyzed with a sophisticated pipeline (Lupton et al. 2001) and have been photometrically (Tucker et al. 2006; Padmanabhan et al. 2008; see also Smith et al. 2002) and astrometrically (Pier et al. 2003) calibrated; the resulting catalog contains almost half a billion distinct detected objects. Well-defined samples of galaxies (Strauss et al. 2002; Eisenstein et al. 2001), quasars (Richards et al. 2002b), stars (Yanny et al. 2009), and other objects are selected for spectroscopy; the survey has obtained roughly 1.8 million spectra of galaxies, stars, and quasars as of Summer 2009.

The principal scientific goal of SDSS-I (2000-2005) and much of SDSS-II (2005-2008) was to create a well-calibrated and contiguous imaging and spectroscopic survey of the northern Galactic cap at high Galactic latitudes, with the spectroscopy primarily focused on extragalactic targets. We refer to this project in what follows as the Legacy Survey. SDSS-II carried out two additional surveys. The Sloan Extension for Galactic Understanding and Exploration (SEGUE; Yanny et al. 2009) imaged a series of stripes sampling low Galactic latitudes (each 2.5 wide and tens to hundreds of degrees long), together with spectroscopy of roughly 250,000 stars, to study Galactic structure, dynamics, and chemical composition. The SDSS Supernova Survey (Frieman et al. 2008) used approximately 80 repeat scans of a $2.5 \times 100^{\circ}$ stripe centered on the celestial equator in the southern Galactic cap to identify Type Ia supernovae with redshifts less than about 0.4 and to use them as cosmological probes (Kessler et al. 2009); almost 500 objects were spectroscopically confirmed as Type Ia supernovae.

These data have been made public in a series of yearly data releases (Stoughton et al. 2002; Abazajian et al. 2003, 2004, 2005; Adelman-McCarthy et al. 2006, 2007, 2008; Abazajian et al. 2009; hereafter the EDR, DR1, DR2, DR3, DR4, DR5, DR6, and DR7 papers, respectively). These data have been used in over 3500 refereed papers to date for studies ranging from asteroids in the solar system to the discovery of the most distant quasars.

It was clear, as SDSS-II was nearing completion, that the wide-field spectroscopic capability of the SDSS telescope and system remained state of the art, and a new collaboration was established to carry out further surveys with this telescope. This new phase, called SDSS-III, consists of four interlocking surveys; it is described in detail in a companion paper (Eisenstein et al. 2011). In brief, these surveys are as follows.

1. SEGUE-2. This survey is an extension of the spectroscopic component of the SEGUE survey of SDSS-II, extending the survey footprint in area and using revised target selection to increase the number of spectra in the distant halo of the Milky Way. SEGUE-2 used the SDSS-I/II spectrograph and ran from 2008 August through 2009 July.

2. The Baryon Oscillation Spectroscopic Survey (BOSS). This survey will measure the baryon oscillation signature in the correlation function of galaxies and the quasar Ly $\alpha$ forest. BOSS started operations in Fall 2009 and consists of a redshift survey over $10,000 \mathrm{deg}^{2}$ of 1.5 million luminous red galaxies to $z \sim 0.7$, together with spectroscopy of 150,000 quasars with $z>2.2$. This has required increasing the imaging footprint of the survey, and we have obtained an additional $\sim 2500 \mathrm{deg}^{2}$ of imaging data in the southern
Galactic cap using the SDSS imaging camera. In addition, in Summer 2009 the SDSS spectrographs underwent a major upgrade (new gratings, new CCDs, and new fibers) to improve their throughput and to increase the number of fibers from 640 to 1000 .

3. The Multi-object APO Radial Velocity Exoplanet Largearea Survey (MARVELS) uses a fiber-fed interferometric spectrograph that can observe 60 objects simultaneously to obtain radial velocities accurate to $10-40 \mathrm{~m} \mathrm{~s}^{-1}$ for stars with $9<V<12$. Each star will be observed roughly 24 times in a search for extrasolar planets. The instrument has been in operation since Fall 2008.

4. The Apache Point Observatory Galactic Evolution Experiment (APOGEE) will use a fiber-fed $H$-band spectrograph with a resolution of 30,000 , capable of observing $300 \mathrm{ob}-$ jects at a time. The spectrograph will see first light in 2011 and will obtain high signal-to-noise ratio $(\mathrm{S} / \mathrm{N})$ spectra of roughly 100,000 stars in a variety of Galactic environments, selected from the Two Micron All Sky Survey (2MASS; Skrutskie et al. 2006).

SDSS-III started operations in 2008 August and will continue through 2014 July. As with SDSS-I/II, the data will periodically be released publicly; this paper describes the first of these releases. For continuity with the previous data releases of SDSS-I/II, we refer to it as the eighth data release, DR8. DR8 includes two significant items of new data relative to DR7.

1. Roughly $2500 \mathrm{deg}^{2}$ of imaging data in the southern Galactic cap, taken as part of BOSS.

2. SEGUE-2 spectroscopy, consisting of 204 unique plates with spectra of roughly 118,000 stars.

As with previous data releases, DR8 is cumulative and includes essentially all data from the previous releases. However, this is not just a repeat of previous data releases, but also an enhancement. In particular, we have re-processed all SDSS-I/II imaging data using a new version of the imaging pipeline with a more sophisticated sky-subtraction algorithm, and all stellar spectra have been re-processed with an improved stellar parameter pipeline.

This paper provides an overview of DR8. Section 2 describes the scope of the imaging and spectroscopic data. More details on the changes to the photometric pipeline and photometric calibration may be found in Section 3, while the spectroscopy, including SEGUE-2 target selection, is described in Section 4. Methods for accessing these data are presented in Section 5. We conclude, and outline the plan for future SDSS-III data releases, in Section 6. The data, and portals to access them, are described in greater detail at the DR8 Web site. ${ }^{73}$

\section{SCOPE OF DR8}

The contents and sky coverage of the data release are summarized in Table 1 and Figure 1. The principal change in the imaging footprint from that in DR7 is the coverage of a large contiguous region, $3172 \mathrm{deg}^{2}$, in the southern Galactic cap. Three disjoint stripes $(76,82$, and 86 , centered roughly at $\alpha=0^{\mathrm{h}}, \delta=-10^{\circ}, 0^{\circ}$, and $+15^{\circ}$, respectively) were included in DR7. The remaining area, roughly $2500 \mathrm{deg}^{2}$, was observed in the Fall and early Winter months of 2008 and 2009; it will be used to identify spectroscopic targets for the BOSS survey. Including the SEGUE stripes, the total area in the southern Galactic cap is $5194 \mathrm{deg}^{2}$.

\footnotetext{
73 http://www.sdss3.org/dr8/
} 
Table 1

Coverage and Contents of DR8

\begin{tabular}{|c|c|}
\hline Imaging & Quantity \\
\hline Total unique imaging area covered & $14,555 \mathrm{deg}^{2}$ \\
\hline Total area imaged, including overlaps ${ }^{\mathrm{a}}$ & $31,637 \mathrm{deg}^{2}$ \\
\hline New imaging area since DR7 & $\sim 2500 \mathrm{deg}^{2}$ \\
\hline Unique objects in database & $469,053,874$ \\
\hline \multicolumn{2}{|l|}{$\begin{array}{r}\text { Spectroscopy } \\
\end{array}$} \\
\hline Spectroscopic footprint area ${ }^{\mathrm{b}}$ & $9274 \mathrm{deg}^{2}$ \\
\hline Legacy & $7966 \mathrm{deg}^{2}$ \\
\hline SEGUE-1 & $1424 \mathrm{deg}^{2}$ \\
\hline SEGUE-2 & $1317 \mathrm{deg}^{2}$ \\
\hline Total number of plate observations $\mathrm{s}^{\mathrm{c}}$ & 2880 \\
\hline Legacy survey plates ${ }^{\mathrm{c}}$ & 1926 \\
\hline Special plates ${ }^{\mathrm{c}}$ & 301 \\
\hline SEGUE-1 survey plates ${ }^{c}$ & 442 \\
\hline SEGUE-2 survey plates ${ }^{\mathrm{c}}$ & 211 \\
\hline Total number of spectra ${ }^{\mathrm{d}}$ & $1,629,129$ \\
\hline Galaxies & 860,836 \\
\hline Quasars & 116,003 \\
\hline Stars & 521,990 \\
\hline Sky & 93,187 \\
\hline Unclassified $^{\mathrm{e}}$ & 37,113 \\
\hline
\end{tabular}

Notes.

${ }^{\mathrm{a}}$ Includes only some of the repeat scans on Stripe 82 taken in 2005-2007 as part of the SDSS Supernova Survey. Roughly 50\% of the SDSS footprint has been imaged more than once.

b This area does not double-count the overlapping footprint of the Legacy and SEGUE surveys.

${ }^{c}$ Each plate has 640 fibers. The number of plates includes some repeat observations.

${ }^{\mathrm{d}}$ Spectral classifications from the idlspec2d code; the totals do not include duplicates or spectra with redshift warning flags. ${ }^{\mathrm{e}}$ That is, objects in which ZWARNING (DR6 paper, Table 4) have any bit other than MANY_OUTLIERS set.

The total sky coverage of DR8 has been calculated more carefully than was done with DR7, so the solid angle coverage of the two cannot be quite directly compared. The figure and the sky coverage numbers do not distinguish some of the "special" scans described in previous data release papers. In particular, the scans covering M31 (DR5 paper), the Orion region (Finkbeiner et al. 2004), and the SEGUE-1 imaging scans (Yanny et al. 2009) are all represented in the figure and are included in the data release along with the Legacy imaging in the same files and database tables.

On the spectroscopic side, the footprint of the survey has increased only slightly, given the small number of SEGUE-2 plates that lie outside the contiguous area of the north Galactic cap (see the red regions in Figure 1). ${ }^{74}$ The numbers of spectra included in various classifications are based on idlspec $2 \mathrm{~d}$ (occasionally referred to as "specBS;" see Section 4.2), one of the two pipelines used in DR7 to classify spectra and determine redshifts. Note that unlike Table 1 in the DR7 paper, this table lists only those unique spectra (i.e., duplicates have been removed), for which idlspec2d gave no redshift warning flags other than MANY_OUTLIERS (see Table 4 of the DR6 paper). Furthermore, the DR7 paper based its numbers on the results of the other of these pipelines, spectro1d (Subbarao et al. 2002), but comparisons of the two pipelines (DR6 paper) show that they are in substantive agreement for over $98 \%$ of spectra.

\footnotetext{
74 The solid angle listed in the DR7 paper for the spectroscopic footprint added together the Legacy and SEGUE-1 areas, double-counting the overlap between the two.
}
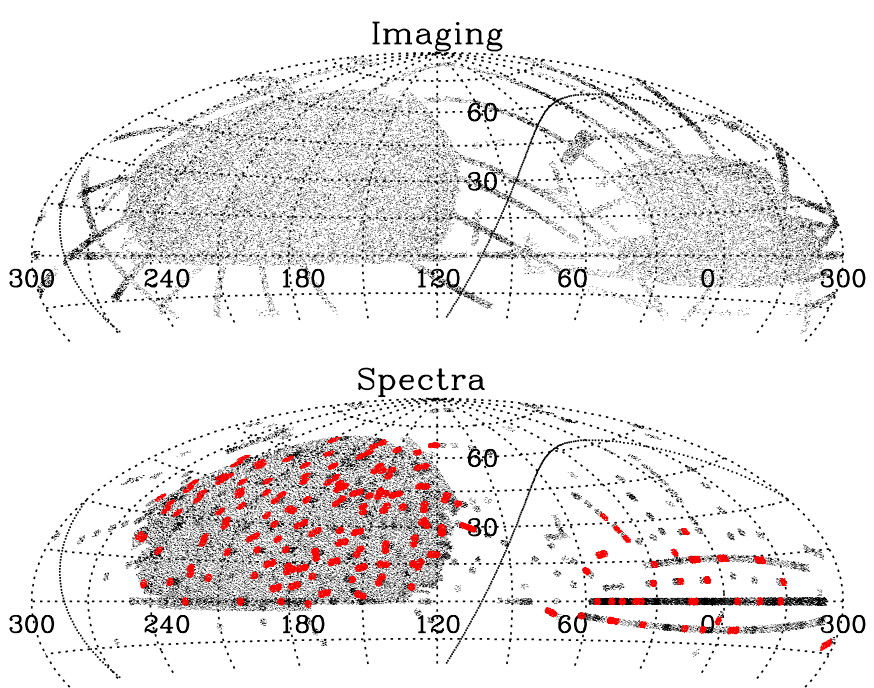

Figure 1. Sky coverage of DR8 in J2000 Equatorial coordinates, in imaging (upper) and spectroscopy (lower). Right ascension $\alpha=120^{\circ}$ is at the center of these plots. The Galactic plane is the solid curve that snakes through the figure. Note the contiguous imaging coverage of the southern Galactic cap (centered roughly at $\alpha=0^{\circ}, \delta=+10^{\circ}$ ); in DR7, this region of sky was covered by a few disjoint stripes. The red regions in the lower panel show the coverage of the SEGUE-2 plates. The BOSS survey will obtain spectra over $10,000 \mathrm{deg}^{2}$, including the contiguous areas in the northern and southern Galactic caps.

The idlspec2d classifications are assigned automatically and do not include the results of any eyeball inspection. This fact, and the absence of a luminosity cut in the definition of quasars, means that the number of quasars differs somewhat from the DR7 Quasar Catalog (Schneider et al. 2010). Objects listed as "unclassifiable" in Table 1 are sources with spectroscopic classification warning flags: most such objects have low $\mathrm{S} / \mathrm{N}$ or problems with the data (e.g., due to bad columns), but this category also includes unusual objects with extreme properties, such as featureless BL Lac objects (e.g., Collinge et al. 2005; Plotkin et al. 2010), extreme broad absorption-line quasars (e.g., Hall et al. 2002), or unusual types of metal-rich or magnetic white dwarfs (e.g., Dufour et al. 2007; Schmidt et al. 2003).

\section{IMAGING DATA}

DR8 includes essentially all the DR7 data, together with the additional data described above. The major exceptions to this statement are as follows.

1. Some of the SEGUE-1 imaging scans described in the DR7 paper pass through the Galactic plane, where the SDSS photometric pipeline does a poor job in regions of very high stellar density. We also processed these fields with software from the Pan-STARRS (Kaiser et al. 2002) collaboration. The results of that analysis are still available on the DR7 Web site, and we do not separately make them available in DR8. However, DR8 does include the SDSS photometric pipeline results in these regions; at sufficiently low latitudes, where the stellar density exceeds 5000 stars deg $^{-2}$ brighter than $r=21$, the SDSS photometry is likely to be unreliable. In particular, there are regions of sky that are so crowded that the software simply times out, and no objects are included in the catalog. This effect is visible in Figure 1 as the discrete scans near $\alpha=300^{\circ}$ that simply fade away at low latitudes.

2. DR8 does not include the co-addition of the repeat scans on Stripe 82 (see the DR7 paper), and it includes only some 
of the Stripe 82 runs (often taken under non-photometric conditions) obtained as part of the SDSS Supernova Survey. In particular, in the resolving (Section 3.4) of Stripe 82, we identified the highest quality run (via the "score" value described in Section 3.4.1) at each position. We include the entire run in DR8 if it is the highest quality at at least one point in the stripe. DR8 includes 118 runs in total on Stripe 82. All 303 Stripe 82 runs are available in DR7, making DR7 the data set to be used for analyses of timevariable phenomena in the stripe.

3. The DR4 paper (see also Ivezić et al. 2004) describes Web sites documenting detailed diagnostics of the photometric and astrometric quality on a run-by-run basis, based both on internal consistency checks and overlaps between adjacent runs. These remain on the DR7 Web site; we have not repeated this analysis for the reprocessing of the imaging data for DR8 or for the new data from Fall 2008 or later. Note that the "ubercalibration" procedure described in Section 3.3 does explicitly report the reproducibility of photometry in overlapping runs. The documentation on the DR8 Web site describes how to check those results.

In the following subsections, we outline further differences to the image processing relative to DR7, including updates to the sky-subtraction algorithm (Sections 3.1 and 3.2), photometric calibration (Section 3.3), resolving overlapping runs (Section 3.4), and astrometric calibration (Section 3.5). We also describe the availability of galaxy morphologies from the Galaxy Zoo collaboration (Section 3.6).

\subsection{Improved Sky Subtraction}

The SDSS imaging data are all processed with the Photometric Pipeline (photo). A number of investigators have shown that the sky-subtraction algorithm used by the DR7 photometric pipeline causes it to systematically underestimate the brightness of large galaxies (Blanton et al. 2005; Lisker et al. 2006; Lauer et al. 2007; Bernardi et al. 2007; West et al. 2010, among others; see also the discussion in the DR4, DR6, and DR7 papers). The sense of the error was to oversubtract the outer regions of large galaxies in the sky estimation, affecting the photometry both of those galaxies and that of smaller and fainter objects in their vicinity. The DR8 imaging data were processed with a more sophisticated sky-subtraction algorithm that reduces this problem, but by no means solves it completely.

Photo estimates the sky level on a rectangular grid of 128 pixels (roughly $50^{\prime \prime}$ ) by calculating the median of the $256 \times 256$ pixels centered on each grid point. The version of photo used in DR7 and earlier data releases simply interpolated bilinearly between these grid points as an estimate of sky; this approach tended to erroneously include light from extended regions around bright galaxies and thus underestimated their fluxes.

The new algorithm adds an additional step of identifying and modeling these extended galaxies before estimating the final sky level. As described in Lupton et al. (2001) and the EDR paper, photo first estimates a single preliminary sky value for an entire $10^{\prime} \times 13^{\prime}$ field. ${ }^{75}$ Using this sky value, it identifies BRIGHT sources ( $>51 \sigma$, corresponding roughly to a star with $r=20$ ). These sources are next run through the deblender to separate overlapping BRIGHT objects. This step is new to this version of photo (before, the deblender was only run after the final

\footnotetext{
${ }^{75}$ For a definition and explanation of the SDSS fields, see the EDR paper.
}

sky model was determined). Models are determined for each child object, and these are then subtracted from the frame. The EDR paper describes the models that are used for galaxies: twodimensional exponential and de Vaucouleurs profiles of arbitrary axis ratio, convolved with the local point-spread function (PSF). As described in the DR2 paper, one can fit the observed profile of galaxies with a linear combination of the best-fit exponential and de Vaucouleurs models to any given galaxy in a given band; we refer to this as the "cmodel." This model is then subtracted from the image, removing the extended wings of the galaxy.

Unsaturated BRIGHT stars are not subtracted at this stage. However, for saturated stars, the outer wings (i.e., outside a radius of 28.'2) are fit to a power law of index $\beta=-3.25$ in ugr $z$ and $\beta=-2.5$ in $i^{76}$; these wings are then subtracted from the image. Now that the wings of bright galaxies and saturated stars have been subtracted, the local sky is estimated as before; that is, a clipped median is measured on a 128 pixel grid and linearly interpolated.

The galaxies (but not the stars ${ }^{77}$ ) are then added back to the sky-subtracted frame, and faint object detection proceeds, as described in the EDR paper. Flags are set to the mask image indicating that a significant part of the sky background at that pixel came from nearby bright objects. If SUBTRACTED is set (flux subtracted is more than $1 \sigma$ above the sky) the pixel is probably trustworthy, while NOTCHECKED pixels (more than $5 \sigma$ above the sky) are probably unreliable (and no further objects will be detected in these regions; the BRIGHT objects will of course be preserved).

With this change in the sky-subtraction routine, the outer parts of galaxies are considerably more extended than they were in the previous version of the software, meaning that they are likely to overlap with more objects in their outer parts. With this in mind, we increase the number of children any blended parent can be decomposed into from 25 to 100 . This has the negative effect of increasing the processing time for fields in which there is a great deal of overlap between objects, such as those at low latitudes and those with bright stars. We find that photo times out on $0.5 \%$ of the fields at $|b|>15^{\circ}$ (45 $\mathrm{deg}^{2}$ in all), almost all of which have a particularly bright star in the field.

\subsubsection{Photometry of Bright Galaxies}

We quantified the accuracy of bright galaxy photometry by adding 1300 artificial galaxies at random positions to SDSS imaging frames, processing them with both the old (DR7) and new (DR8) versions of photo, and comparing the results with the true input values. The simulated galaxies, which have Sérsic radial profiles with a range of inclinations and Sérsic indices, follow the observed correlation between apparent magnitude and angular size seen for real galaxies (Figure 2). However, we biased the sample somewhat to larger and brighter objects, as this is the regime in which the sky-subtraction errors are likely to be worst. In addition, the sample is approximately size limited at a Petrosian (1976) half-light radius $r_{50} \sim 5^{\prime \prime}$.

The results are shown in Figure 3, where we plot the difference between measured and true half-light radii and magnitudes in the $r$ band for the simulated galaxies in the DR7 (red) and DR8 (blue) versions of the pipeline. Results for the other bands are similar. The new sky-subtraction algorithm improves things

\footnotetext{
76 A small fraction of the photons scatter within the thick chips used in the $i$ band, yielding an extended halo around stars.

77 Not adding the stars back in greatly simplifies the deblending around bright stars, which otherwise cause significant parts of the frame to blend into a single object.
} 


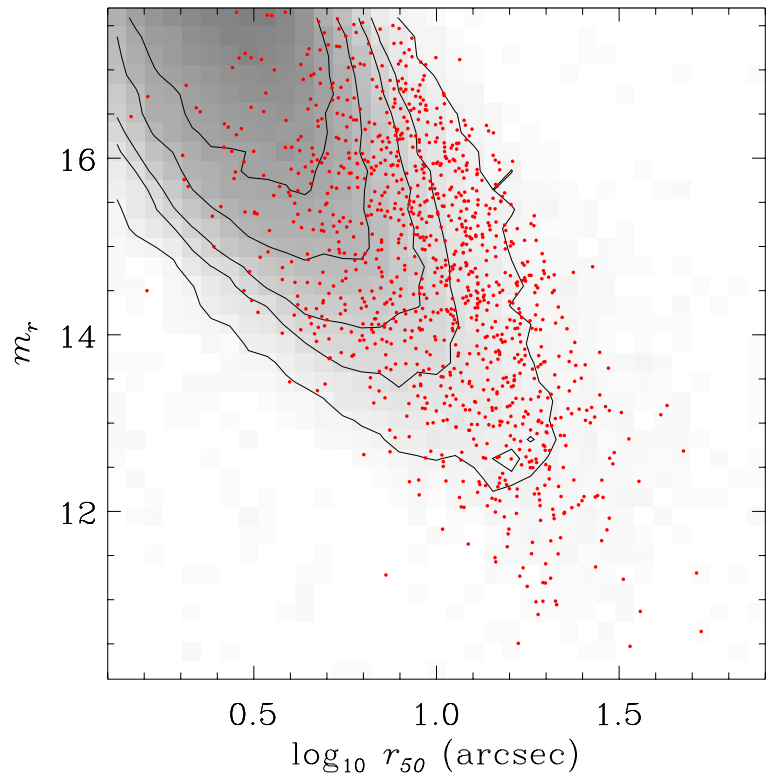

Figure 2. Gray scale and contours show the distribution of galaxies in SDSS in apparent magnitude and Petrosian half-light radius. The red dots show the distribution of artificial galaxies added to the imaging frames to explore the ability of the pipeline to photometer large galaxies. We have deliberately biased the sample of artificial galaxies to larger objects at a given magnitude.

somewhat, but is not a panacea. The principal trend is with galaxy area, because it is the quantity that couples most directly to the sky measurement. The improvement is subtle at best and is only visible for galaxies with $r_{50}>30^{\prime \prime}$. The roughly $1 \mathrm{mag}$ of bias at $r_{50} \sim 50^{\prime \prime}$ is reduced in the DR8 pipeline by only about $0.25 \mathrm{mag}$. Additionally, there is a distinct bias in the measured sizes themselves, which is similar in the two pipelines. Some of the problem may not be due to sky subtraction, but rather to the deblender systematically assigning some of the light in the outer parts of galaxies to superposed fainter stars and galaxies.

\subsubsection{Photometry of Faint Galaxies Near Bright Galaxies}

A related problem reported by Mandelbaum et al. (2005) is that the previous sky-subtraction procedure suppressed the number density of faint galaxies around bright galaxies and distorted the measured shapes of these faint galaxies, which affects measurements of galaxy-galaxy lensing and the clustering of faint objects near bright objects. We here examine the suppression in the number density, comparing the DR7 and DR8 pipelines. Figure 4 compares the number density of faint galaxies relative to the mean in the two versions of the pipeline, as a function of the angular distance from bright galaxies.

The upper panels and lower left panel of Figure 4 show a test that used a common set of foreground galaxies $\left(12<r_{\text {model }}<\right.$ 18 ), divided into magnitude bins. The faint galaxies came from the original catalog of source galaxies in Mandelbaum et al. (2005), which included well-resolved galaxies with $r<21.8$ selected from the DR7 reductions. For DR8, we selected a similar catalog of source galaxies described in R. Reyes et al. (2011, in preparation). In these panels, we did not attempt to exclude source galaxies that are physically associated with the lens, which means that we expect some increase in the number density at small scales where galaxy clustering is important. Additional effects that should modify the number density include deblending errors around the bright galaxy,

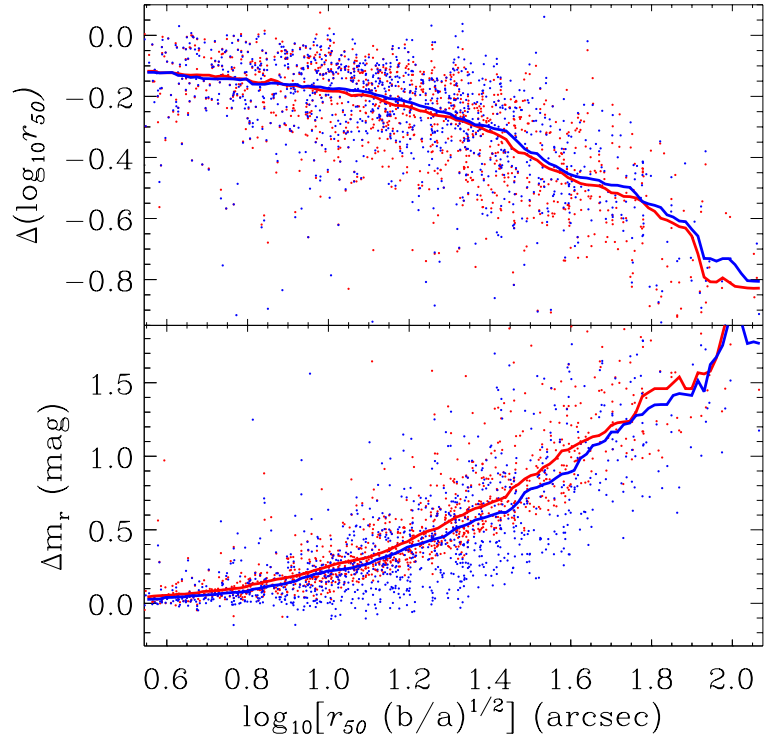

Figure 3. Differences between the true and measured $r$-band half-light radii and magnitudes as a function of $r_{50} \times(b / a)^{1 / 2}$ (whose square is proportional to the area of the galaxy; here $b / a$ is the axis ratio of the galaxy from the model fit), for a sample of simulated galaxies. The sample has Sérsic profiles, with a range of magnitudes and sizes (and therefore surface brightnesses), designed to sample the observed distribution of large bright galaxies. The measured magnitudes are the combined "cmodel" magnitudes using the exponential and de Vaucouleurs fits, and the measured sizes are the effective radii from the better of those two fits for each galaxy. Top panel shows the logarithmic difference between the measured half-light radius and the true one $\left(\Delta \log _{10} r_{50}=\right.$ $\left.\log _{10} r_{50 \text {,meas }}-\log _{10} r_{50, \text { true }}\right)$. Bottom panel shows the magnitude difference $\left(\Delta m=m_{\text {meas }}-m_{\text {true }}\right)$. Results are shown both for the version of photo used in DR7 (red) and DR8 (blue). The running median values as function of radius are shown as the solid lines. The new code reduces the bias at large area, but only incrementally.

gravitational magnification, ${ }^{78}$ and dust extinction (which tends to counteract magnification but appears to be weaker for lowredshift galaxies; Ménard et al. 2010).

As shown in the three aforementioned panels of Figure 4, the number density of faint galaxies around bright foreground galaxies is strongly affected by the foreground at angular separations less than 100". The $12<r<15$ galaxies have such a large angular extent that the number density is severely suppressed below 50". The sky mis-estimation near $15<r<17$ galaxies causes a $\sim 5 \%$ suppression in the number density for $30^{\prime \prime}<\theta<90^{\prime \prime}$. Finally, for the $17<r<18$ foreground galaxies, the predominant effect in the source number density is clustering, but there is a subtle effect around $50^{\prime \prime}$ that is likely due to sky mis-estimation. In all three panels, the curves for the previous reductions exhibit a significant bump around 20", the origin of which is unclear. This bump is present around stars as well (for which lenssource clustering is not a possible explanation), but in the DR8 reductions, the bump goes away almost completely for both stars and galaxies. The disappearance of this artifact at $20^{\prime \prime}$ constitutes a substantial improvement in the new pipeline. Unfortunately, the suppression in source counts from $40^{\prime \prime}<\theta<90^{\prime \prime}$ has improved only slightly.

The lower right panel in Figure 4 shows the results of a different test, using the new source catalog from the DR8 reductions only. For this catalog, we have generated photometric

\footnotetext{
78 Based on the lensing shear that is measured and the slope of the number counts of the source sample, we anticipate an effect that is at most $3 \%$ at $10^{\prime \prime}$, is strictly positive, and decreases with scale.
} 


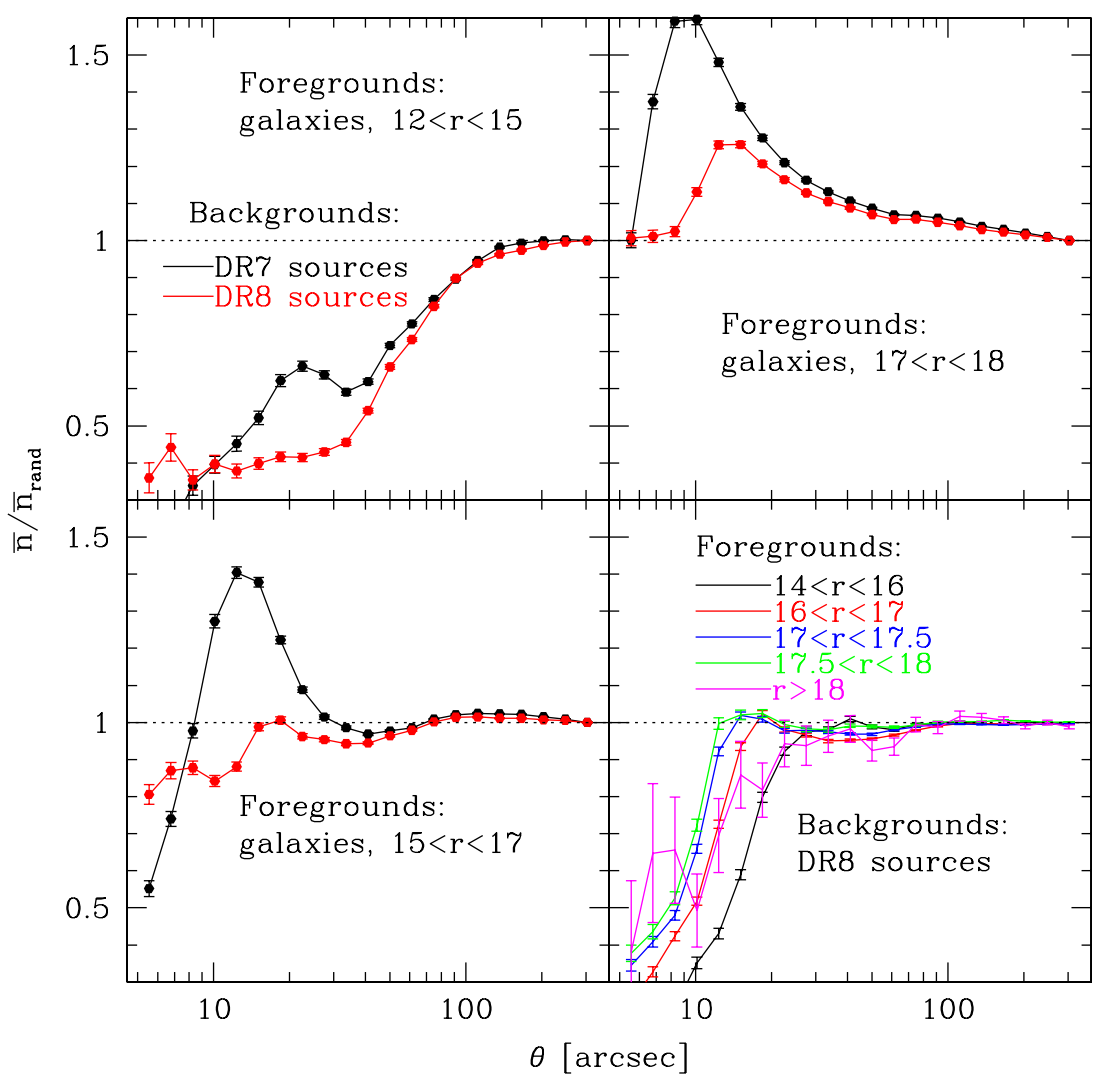

Figure 4. Top left, bottom left, top right: number density of source galaxies as a function of distance from bright foreground galaxies. Each panel is a separate foreground magnitude bin as labeled on the plot. The black solid and red dashed lines show the results for DR7 and DR8, respectively. Bottom right: same as other panels but for DR8 only, where separate line colors and styles indicate different foreground magnitude bins. In this case, unlike for the other panels, source galaxy photometric redshifts were used to exclude sources that are in front of, or are physically associated with, the foreground object.

redshifts for all sources using ZEBRA (Feldmann et al. 2006; R. Nakajima et al. 2011, in preparation); these photometric redshifts were used to isolate sources at $z>0.3$, thus eliminating almost completely the correlations between foregrounds and sources due to galaxy clustering. The remaining effects in the number density are due to sky subtraction, gravitational magnification, dust extinction, and possibly a very low level $(<2 \%)$ of clustering due to catastrophic photo- $z$ errors. As shown here, the sky subtraction suppresses the source number density by $\sim 4 \%$ for $30^{\prime \prime}<\theta<90^{\prime \prime}$. Note that extended dust halos around galaxies (Ménard et al. 2010) cannot be the explanation of the effect, as the suppression is seen around stars (not shown) as well as galaxies.

The magnitude of the galaxy number suppression depends not just on the properties of the bright foreground galaxy (as illustrated in this figure), but also on the properties of the fainter nearby galaxies, with fainter or lower surface brightness galaxies being more severely affected. Position on the CCD is also a factor: near the edges of the fields, the sky level must be extrapolated, which means that sky estimates are worse within 256 pixels of the edge.

\subsection{Improved Sky Subtraction in Post-processing}

DR8 includes "corrected frames," FITS files of each frame which have been bias subtracted and flat fielded, with bad columns and cosmic rays interpolated over. Each frame has a World Coordinate System (WCS) giving the full astrometric solution in its header, and the pixel values are calibrated to fluxes. Thus, astrometry and photometry can be performed directly on the image. These images have also been sky subtracted, using an algorithm that goes beyond the one we have described above. But the photometric pipeline has not been run on these corrected frames, as we implemented this fix after the processing of the bulk of the data was completed, thus these improvements are not reflected in the object catalogs.

Our method treats each run as a whole and fits a smooth function to the variation of the sky background using a heavily masked and binned image of the run. The method is described in full by Blanton et al. (2011). We find good agreement within the typical image noise between the photometry of point sources in these images and the SDSS catalog.

More critically, we have also tested the effect of our background subtraction on the photometry of large galaxies by inserting fake galaxies into the raw data and measuring their properties after background subtraction. We find that this sky-subtraction technique introduces biases of $>0.1$ mag only at half-light radii $r_{50}>100^{\prime \prime}$. For typical large galaxies, our results agree at the $5 \%$ level with those of the Montage package distributed by the NASA/IPAC Infrared Science Archive, which uses overlapping observations from adjacent runs to determine the sky levels (Berriman et al. 2003). However, any actual photometry of such galaxies is much more difficult, requiring very accurate deblending as well to achieve unbiased results. These issues are more fully explored in Blanton et al. (2011). See the paper by West et al. (2010) for another approach to the problem.

We recommend these sky-subtracted images as a robust starting point for users interested in reprocessing SDSS images. Note that for very large systems (for example, for intracluster 
light studies) there may still be biases present. For this reason, the corrected frames also contain the information needed to undo the global sky subtraction.

\subsection{Photometric Calibration}

In SDSS-I/II, the default photometric calibration method used an auxiliary 24 inch telescope (the "Photometric Telescope," hereafter PT), which observed a set of standard stars (Smith et al. 2002) to determine the photometricity and extinction coefficients for each night, as well as a large set of calibration fields on the stripes observed by the $2.5 \mathrm{~m}$ to place them on a uniform photometric system (Tucker et al. 2006). While this approach allowed us to reach our goal of $2 \% \mathrm{rms}$ photometric calibration in all bands (Ivezić et al. 2004), it was limited by concerns about the slightly different photometric systems of the PT, the $2.5 \mathrm{~m}$, and the Naval Observatory $1.0 \mathrm{~m}$ telescope in Flagstaff, where the standard stars were initially put onto a common system. In addition, this approach did not take advantage of the overlap between adjacent scans.

An alternative approach, called "ubercalibration" (Padmanabhan et al. 2008), is a purely internal calibration using only the overlaps between adjacent scans of the $2.5 \mathrm{~m}$. This new calibration is forced to be on the same zero point (within $1 \mathrm{mmag}$ in griz and $3 \mathrm{mmag}$ in $u$ ) on average as the DR7 calibration, but it does not use any data from the PT. As described in Padmanabhan et al. (2008), the calibration has residual errors of order $1 \%$ in griz and $2 \%$ in $u$.

Ubercalibration uses a series of scans running perpendicular to the main survey runs, performed in a fast, binned mode available on the SDSS camera, referred to as the Apache Wheel scans. The uncalibrated version of these data and their associated reductions are available as flat files on the DR8 Web site, but their proper use requires a great deal of care.

We made both PT calibration and ubercalibration results available in DR6 and DR7, but with DR8, we release only the results based on the ubercalibration. In particular, the PT calibration was not performed for the new imaging data. The DR7 ubercalibration process used a different flat-field scheme from that used in DR8; this difference dominates the difference in the calibration between the two.

Schlafly et al. (2010) have used DR8 photometry to study the effects of Galactic reddening on star colors (in particular, the blue tip of the stellar locus); they find rms spatial variations in these colors of $18,12,7$, and 8 mmag in $u-g, g-r, r-i$, and $i-z$, respectively. These variations include possible contributions from stellar population variations and errors in the Schlegel et al. (1998, hereafter SFD) dust map as well as photometric calibration errors and so represent upper limits on the amplitude of the latter. Of course, these values are consistent with the $1 \%$ rms calibration errors quoted above in $g, r, i$, and $z$. Schlafly et al. (2010) also find systematic differences in zero points between the north and south Galactic cap of $8,22,7$, and 12 mmag in $u-g, g-r, r-i$, and $i-z$, respectively (as Figure 1 shows, the north and south are tied together photometrically with a few SEGUE imaging scans). Again, these differences may be due in part to errors in the SFD map and stellar population differences.

With the changes in photo and calibration, it is interesting to compare the DR7 and DR8 photometry. For a sample of $18<r<19.5$ stars randomly selected over the DR7 footprint, we found the PSF magnitudes to differ by an rms of 11-14 mmag in griz. In the $u$ band, we further restricted ourselves to $u<20$ and found rms differences of $20 \mathrm{mmag}$.

\subsection{Resolving the Imaging}

The SDSS imaging camera (Gunn et al. 1998) observes the sky in six parallel scanlines, each $13^{\prime}$ wide and as much as hundreds of degrees long. As is discussed in detail in the EDR paper, the way the camera scans the sky produces quite a bit of overlap between the scanlines. The geometry of the great circles of the main SDSS survey naturally gives rise to substantial overlap at the ends of the stripes (York et al. 2000); it is this overlap which allows the photometry of the scans to be tied together (Section 3.3). The overlap also allows accurate photometry of objects which may be close to a CCD edge in one imaging run but far enough away to allow proper measurement in the adjacent run. Roughly $50 \%$ of the SDSS imaging footprint was observed more than once, and the first two entries in Table 1 show that because of the overlaps, the total area imaged is more than double the unique area.

However, for statistical studies, one needs a single unique detection of each object in the sky, which requires that we resolve the overlaps, identifying a single imaging run to represent each point in the SDSS imaging footprint. In previous data releases, this was done by simply bisecting the overlap between adjacent scanlines; the primary detections of all objects lying on one side of the bisector were assigned to one scanline, and those on the other side were assigned to the other. This procedure has several disadvantages that motivated us to revisit the problem.

1. This approach makes most sense when the scans are all roughly parallel great circles, in the $\lambda, \eta$ coordinate system used by the Legacy survey (EDR paper; Pier et al. 2003). It does not translate well to scans that use a different survey pole, such as the SEGUE imaging scans, the so-called oblique scans, and others.

2. Because of the focus on the Legacy survey in SDSS-I/II, the resolution of the scans made reference to the boundaries of an ellipse on the sky into which the northern Galactic cap scans approximately fit (York et al. 2000). This meant that scans that happened to fall outside that ellipse were not flagged as "primary" (see below).

3. Anticipating the possibility of further scanlines beyond the boundaries of the existing imaging runs, the resolve algorithm applied the bisector line (and thus flagged perfectly good imaging data as "secondary;" see below) at the boundaries of the survey.

In this section, we describe the new resolve algorithm. We first determine the geometrical sky coverage of the survey (or "window function," which describes which imaging data are primary at each point of the survey footprint) then resolve it to produce a catalog of primary, unique detections of objects. The primary area of the survey is constructed as a union of the individual SDSS fields, with the highest scoring field covering any given point of the sky (in the sense described in Section 3.4.1) labeled as "primary." We will refer to detections in a non-primary part of a field as "secondary" if they are associated with a primary detection. If detections in non-primary areas are not associated with any primary detection, we refer to them as "best." Variable objects or those close to the photometric limit of the catalog can give rise to such unique detections in secondary observations of a given field.

\subsubsection{Scoring Each Field}

As described in the EDR paper, the individual scanlines are divided into $10^{\prime} \times 13^{\prime}$ fields (1489 pixels by 2048 pixels), 
with 128 pixels of overlap between them. The photometric pipeline analyzes each field separately. As a first step in defining the geometry of the full survey, we trim 64 pixels (about $25^{\prime \prime}$ ) off each edge of the field. This removes the overlap between adjacent fields along the scanline, while the trimming perpendicular to the drift-scan direction prevents the primary catalog from including objects that are too close to the frame edge to be properly measured.

Each point on the sky can be covered by one or more fields, and we need to identify the best of these to be the primary detection at this point. We do so by first ranking the fields according to a metric which we refer to as its "score." This score is based on the $r$-band seeing, the sky brightness in $r$, the measurement of photometricity from ubercalibration and the APO $10 \mu \mathrm{m}$ cloud camera (Hogg et al. 2001), and any indications of problems when the imaging data were taken (poor focus or tracking, unusually high CCD noise or evidence that the flat-field petals were not properly opened during the observations). Each field is given a numerical score between 0 and 1 ; values below 0.6 indicate that the data are not photometric (as determined by the ubercalibration process itself and by the cloud camera). These scores are used in what follows to define the primary field covering each point on the sky.

\subsubsection{Defining the Window Function}

The primary survey area is defined as the union of all the fields. Determining the window function requires identifying the fields that cover each position on the sky, and deciding which of those fields should be considered primary at that position.

We treat each field as a rectangle on the sky defined by its trimmed area as described above. There is a unique set of disjoint polygons (hereafter "balkans") on the sky defined by all the field boundaries, which are calculated using the mangle package of Swanson et al. (2008). Each field is divided into one or more balkans, and each balkan is fully covered by a unique combination of one or more fields.

We assign the primary field associated with each balkan as follows. We start with the highest scored field overall and call it primary for all the balkans covered by it. Then we step to its adjacent fields in the same scanline. As long as their score is within 0.05 of the initial field, we consider them to be primary for the balkans they cover as well; this avoids switching field by field between two comparably good runs on the same scanline. We continue along the scanline in both directions until we reach a substantially worse field than the first (i.e., a decrease in score of >0.05). When that happens, we step to the next highest ranked field that has not already been assigned and execute the same steps for that field. Of course, if a balkan has already been assigned a primary field, that assignment is not changed. This process is iterated until we have assigned all of the fields in the survey.

\subsubsection{Resolving Catalog Detections}

Once the window function is defined, we can resolve multiple detections of individual objects. Each detection of an object has an associated flag, resolveStatus, that reports the results of this procedure. This exercise is performed only for those objects that are not parents of deblended children, are not classified as BRIGHT detections (because they will be remeasured in a second pass through the pipeline; see the EDR paper), and have not been classified as SKY (blank fields at which spectroscopic fibers can measure the spectrum of the sky) or CR (cosmic rays). We select objects which are in the full area of each field, excepting the
64 rows at the top and bottom (that is, those overlapping adjacent fields on the same scanline). Along those edges in the driftscan direction, we take care to account for small astrometric differences that might give rise to lost or duplicate objects: if any two detections in adjacent fields are within $2^{\prime \prime}$ of each other and straddle an edge, one and only one of them is chosen as primary for the run. The RUN_PRIMARY bit of resolveStatus is set for those objects that pass this cut.

We next define the "survey primary" detections, unique detections among all the imaging runs. In order to allow for small astrometric jitter between adjacent balkans, we select RUN_PRIMARY detections that are within the trimmed area of the primary field covering the balkan, or within $1^{\prime \prime}$ of the edge of the balkan, and match each selected detection to the current list of primary detections. If it matches a previous primary detection, as it might if it is near the edge of the balkan, then it is not included; otherwise, it is assigned SURVEY_PRIMARY in resolveStatus.

This process has the potential to miss some transient or low $\mathrm{S} / \mathrm{N}$ sources, which may be detected in some fields covering a region of sky but not in the primary field. To identify these, we loop over all the fields and match all the RUN_PRIMARY objects to the full list of SURVEY_PRIMARY objects. Objects that are unmatched are good detections in this field, but have no corresponding primary objects, and so fall into a separate category; we label them as SURVEY_BEST in resolveStatus.

Finally, the duplicate detections of primary or best objects are called "survey secondary" detections. To find these cases, we loop over all fields and select objects which are RUN_PRIMARY but neither SURVEY_PRIMARY nor SURVEY_BEST. We match these objects against the SURVEY_PRIMARY and SURVEY_BEST lists from the other fields. If the detection is matched, and the balkan containing the primary/best observation contains the current field we are considering, then this detection is labeled SURVEY_SECONDARY.

This process produces a list of all of the primary, best and secondary detections. In addition, for each secondary detection we know which primary or best detection matches it. The documentation on the DR8 Web site describes how to use this information, which is useful for finding multiple observations of the same object.

\subsection{Differences in Astrometric Calibration}

The quality of the DR8 astrometry unfortunately is degraded from that in DR7 due to a number of software errors introduced in the DR8 reprocessing. The following effects apply to the DR8 astrometry.

1. Color terms were not included in the transformation from position on the detector to right ascension and declination. This causes 10-20 mas systematic errors with color in catalog positions. Systematic errors of similar size are introduced in the measure of position offsets between filters; the errors are somewhat smaller in $i$ and $z$, and somewhat larger in $u$ and $g$.

2. The DR7 astrometry was calibrated against the Second US Naval Observatory CCD Astrograph Catalog (UCAC2; Zacharias et al. 2004). The UCAC2 positions were propagated to the SDSS epoch using proper motions from UCAC2 for declinations below $41^{\circ}$. Because UCAC2 proper motions at high declinations were not available, SDSS+USNO-B (Monet et al. 2003) proper motions (Munn et al. 2004) were used for higher declinations. In DR8, the $\mathrm{UCAC} 2$ proper motions in right ascension were incorrectly 
applied, introducing systematic errors in right ascension of 5-10 mas. For declinations above $41^{\circ}$, the SDSS+USNO-B proper motions were not applied at all, introducing systematic errors in both right ascension and declination of typically 20-40 mas, and as high as 60 mas.

3. Previous SDSS data releases based the catalog right ascension and declination values on the catalog objc_rowc and objc_colc frame coordinates. These coordinates use the $r$-band centroid for unsaturated stars brighter than $r=22.5$, but for stars that are saturated in the $r$ filter but unsaturated in another filter, or fainter than 22.5 in $r$ but better exposed in another filter, use the centroid from an optimal filter. For DR8, the right ascension and declination values use the $r$-band centroid for all stars. This increases the statistical error for some stars fainter than $r=22.5$ over that in earlier data releases. For stars saturated in $r$ but unsaturated in other filters, it can introduce systematic errors of up to 100 mas compared to previous data releases.

The systematic errors introduced in DR8 are typically smaller than or comparable to the 45 mas systematic errors that characterize the SDSS astrometry for brighter stars. Given these problems (which we plan to fix in a future data release), we recommend that users interested in precise astrometry, especially statistical studies of star positions at the $<0$ '. 1 level, use the DR7 results. For most applications, however, the quoted positions should be acceptable. Note in particular that the proper motions tabulated in the Catalog Archive Server (CAS) are only mildly affected by these problems, as the systematic errors in position largely cancel when calculating the proper motions. The primary effects on the proper motions are to introduce an

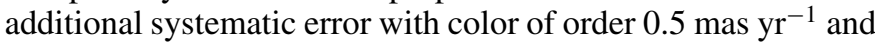
to introduce an additional source of statistical error (in right ascension only) for stars with $\delta>+41^{\circ}$ of order 1 mas $\mathrm{yr}^{-1}$.

\subsection{Galaxy Zoo}

Galaxy Zoo is a Web-based project ${ }^{79}$ that used the collective efforts of hundreds of thousands of volunteers to produce morphological classifications of galaxies. In the first phase of Galaxy Zoo, about 100,000 volunteers visually inspected gri color composite images of galaxies in the SDSS Main Galaxy spectroscopic sample (Strauss et al. 2002) and classified them as ellipticals, spirals, mergers, or star/don't know/artifact. In this phase, the project obtained more than $4 \times 10^{7}$ unique classifications. These basic classifications are consistent with those made by professional astronomers on sub-sets of SDSS galaxies (e.g., they agree $90 \%$ of the time with Fukugita et al. 2007), thus demonstrating that the data provide a robust morphological catalog. Full details on the classification process, including the operation of the site, are given in Lintott et al. (2008).

The initial Galaxy Zoo data containing the basic classification data for 667,945 Main Galaxy sample galaxies (having measured redshifts in the range $0.001<z<0.25$ and clean $u$ and $r$ photometry in SDSS DR7) have recently been made public (Lintott et al. 2011). For each galaxy, this catalog includes weighted counts of volunteer "votes" for the elliptical galaxy, spiral galaxy (split into clockwise or anticlockwise arms and edge-on/arms not visible), merger and "star/don't know/artifact" categories. In addition, the catalog also includes votes corrected for perception bias effects and information on confidence levels of the classification. Those galaxies whose

\footnotetext{
79 http://www.galaxyzoo.org
}

debiased votes give an unambiguous answer $(>80 \%)$ for their morphology are explicitly labeled as elliptical or spiral. Full details are given in Lintott et al. (2011). These initial Galaxy Zoo classifications are included in DR8, accessible through the CAS (Section 5). The resulting catalog provides basic morphological classifications from visual inspection alone, providing an alternative to classifications based on parameters such as color, concentration, or structural parameters.

\section{SPECTROSCOPIC DATA}

The principal changes in the spectroscopic data from those available in DR7 are as follows.

1. The inclusion of 211 new plates with spectroscopy of 118,000 stars, from the SEGUE-2 survey (Section 4.1).

2. Improvements in the SEGUE Stellar Parameter Pipeline (SSPP; Section 4.4).

3. Improved data quality diagnostics on all plates (Section 4.5).

4. The release of 108 spectroscopic plates observed before Summer 2008 which were not included in DR7 and improved processing of a number of plates that targeted open and globular clusters used for SEGUE calibration (Section 4.6).

5. Improved matching between the photometric and spectroscopic objects in the CAS (Section 4.7).

In addition, the redshifts and classifications included in DR8 are now based on idlspec2d instead of spectro1d (Section 4.2), and we make available the results of an independent code to measure galaxy emission-line strengths and other quantities derived from galaxy spectra (Section 4.3).

\subsection{SEGUE-2 Target Selection}

The SEGUE-1 paper (Yanny et al. 2009) describes how that survey selected spectroscopic targets from extreme metal-poor star candidates to low-mass stars to F-star tracers of the Galactic halo potential. For SEGUE-2, these selection algorithms were refined in various ways, as detailed in C. Rockosi et al. (2011, in preparation; see also Eisenstein et al. 2011). We summarize the differences between SEGUE-1 and SEGUE-2 here.

In SEGUE-1, there were two pointings of 640 spectra on each $7 \mathrm{deg}^{2}$ plate area on the sky (hereafter a "tile"), one consisting of a relatively short exposure on bright stars, and the other a longer exposure on fainter stars. The magnitude split between the bright and faint plates was at $r=17.8$ for $g-r<+0.55$ and $r=17$ for $g-r>+0.55$, allowing better $\mathrm{S} / \mathrm{N}$ in the blue for cool stars. The $\mathrm{S} / \mathrm{N}$ for stars as faint as $g=19.5$ was adequate to determine abundances using the SSPP. SEGUE-2 focused on spectroscopy of stars in the distant halo and observed a single long-exposure pointing of 640 spectra on each tile, allowing it to cover more sky in the year of the survey. Fifty percent of the stars with SEGUE-2 spectra have $17.4<g<18.9$, and the median $\mathrm{S} / \mathrm{N}$ per $\AA$ of the SEGUE-2 spectra is 33.1. For comparison, $50 \%$ of the SEGUE-1 spectra have $16.5<g<18.9$, and the median S/N per $\AA$ is 26.0. A total of 211 observations were made of 204 pointings in SEGUE-2, as shown in Figure 1.

All the targets were selected using the SDSS imaging data and recalibrated SDSS+USNO-B proper motions (Munn et al. 2004) from DR7. Plates from Fall 2008 were designed using a preliminary version of the DR7 data because the final version was not yet ready. In order that the survey target selection be reproducible, the photometry and astrometry for all objects 
within the area of each plate, available at the time the plates were designed, are included in a separate table in the DR8 database.

SEGUE-2 increased the fraction of fibers devoted to candidate objects in the outer halo over that in SEGUE-1 and modified the selection criteria for red giant branch (RGB) stars and blue horizontal branch stars in order to increase the number of high-quality spectra for these categories. There were three target selection categories in SEGUE-1, the F/G, G, and dK,dM categories, which accounted for over half the 240,000 SEGUE-1 targets. These were dominated by nearby main-sequence stars, mostly in the disk, because they used only a simple color and magnitude cut. Because SEGUE-2 observed about half the number of stars per tile as SEGUE-1, we devoted only 100 fibers per plate to a similar category called MS turnoff stars. The SEGUE-2 turnoff stars are selected as targets with $18<g<19.5,+0.10<g-r<+0.48$, and range in distance from 6 to $13 \mathrm{kpc}$.

The SEGUE-2 selection of stars on the RGB was improved and extended to cooler giants based on the results from SEGUE-1. A total of 150 fibers per plate was devoted to this category. As in SEGUE-1, the selection required that the recalibrated USNO-B+SDSS proper motions be consistent with 0 at $3 \sigma$ to isolate distant objects. The confirmed low-gravity RGB stars from SEGUE-1 as well as the globular and open cluster fiducial sequences from An et al. (2008) and Clem et al. (2008) were used to identify regions of the $u-g, g-r$ color-color diagram where late $\mathrm{K}$ and $\mathrm{M}$ giants are easily separated from the stellar locus. The SEGUE-2 targeting also improved on the SEGUE-1 selection of warmer RGB stars using the $l$-color (Lenz et al. 1998) indicator of low-metallicity and (to a lesser extent) low-gravity stars.

The SEGUE-2 ugr color selection of blue horizontal branch stars includes only stars blueward of the old main-sequence turnoff, $g-r<+0.05$. SEGUE-2 allocated as many as 100 fibers per plate to such stars, but filled all those fibers only in the most crowded fields. The fact that the density of blue horizontal branch stars and cool red giant candidates was low was a major motivation to obtain only one tile per pointing and to maximize the area of SEGUE-2.

New to SEGUE-2 are spectra of candidate old, metal-rich hypervelocity stars using the color and proper motion selection criteria described in Kollmeier et al. (2010). In addition, 50 fibers per plate were allocated to high velocity candidates with a $g-r$ color close to that of the main-sequence turnoff and velocities (based on proper motions) estimated to be at least $3 \sigma$ above $300 \mathrm{~km} \mathrm{~s}^{-1}$. Finally, the selection of cool subdwarf and low-metallicity stars was adjusted for improved efficiency based on the results of searches for those objects using SEGUE-1 and SDSS spectra (Lépine \& Scholz 2008).

SEGUE-1 and SEGUE-2 spectroscopy was performed on only a small fraction of the SDSS footprint, but both the SEGUE-1 and SEGUE-2 target selection algorithms were applied to all the available imaging data; these results are included in the DR8 database (Section 5), as they may be of use for statistical studies of the spatial distribution of various populations of stars.

\subsection{Spectroscopic Classification and Redshift Measurement}

The SDSS spectra are classified as stars, galaxies, or quasars, and redshifts are determined with an automated routine. As the DR6 paper describes, this was done using two independent pipelines, one (spectro1d) which worked by crosscorrelation with a family of templates, and emission-line fits, followed by eyeball inspection of problematic cases, and another (idlspec2d or specBS) which does direct $\chi^{2}$ fitting of templates to the spectra. In DR8, we only make the latter available; as described in the DR6 paper, the two pipelines give substantially the same results for over $98 \%$ of spectra. The idlspec $2 \mathrm{~d}$ pipeline has not been properly described in print before, so we do so here.

The classification and redshift-fitting procedures described below use the spectrum and associated error estimate vectors (in the form of inverse variances) to derive parameters of interest through $\chi^{2}$ model fitting to the spectra in pixel space (see Glazebrook et al. 1998 for an early version of this approach).

A "skymask" is constructed and used to give zero weight in the fit to pixels that show either bad sky subtraction in one of the 15 minute exposures contributing to a given observation of a plate or extremely high relative sky brightness in all exposures. The condition of bad sky subtraction applies to all object spectra at a given wavelength: we divide all sky-subtracted sky spectra on a given plate by their associated error vectors, square these scaled values, and mask wavelengths at which the 67 th-percentile value of the resulting quantity exceeds 3 . Bright sky is defined on an object-by-object basis wherever the skyline brightness exceeds the sum of the extracted object flux plus 10 times its associated error. The skymask defined by these two conditions is grown by two extracted pixels in either direction. Regions of spectra affected by bad CCD pixels or by excessive cosmic-ray hits are given an inverse variance of zero by the twodimensional extraction software routines and are not explicitly flagged in the redshifting and classification analysis.

Spectroscopic redshift determination and object classification is done for all spectra without regard to the category by which they were targeted for spectroscopy, using four separate spectral template classes: galaxies, quasars, stars, and cataclysmic variable stars.

The galaxy class is defined by a rest-frame principalcomponent analysis (PCA) of 480 main sample galaxies (Strauss et al. 2002) observed early in the SDSS, which is used to define a basis of four "eigenspectra" corresponding to the four most significant modes of variation in the PCA analysis. The redshifts of the galaxy PCA training sample are established by fitting each spectrum with a linear combination of two stellar template spectra and a set of narrow Gaussian profiles at the wavelengths of common nebular emission lines. The stellar template spectra used in this procedure are obtained from the first two components of a PCA analysis of 10 velocity standard stars in M67 observed by SDSS (plate 321, observed on Modified Julian Date (MJD) 51612). The galaxy PCA training sample redshifts were verified by visual inspection.

For all spectra, a range of trial galaxy redshifts is explored from $z=-0.01$ to $z=1.00$ with a separation of $138 \mathrm{~km} \mathrm{~s}^{-1}$ (i.e., two pixels in the reduced spectra). At each trial redshift, the galaxy eigenbasis is shifted accordingly, and the error-weighted data spectrum is modeled as a minimum $-\chi^{2}$ linear combination of the redshifted eigenspectra and a quadratic polynomial to absorb low-order calibration uncertainties. The $\chi^{2}$ value for this trial redshift is stored, and the analysis proceeds to the next trial redshift. This procedure is facilitated by the constantvelocity (constant log-wavelength) pixel width of the reduced SDSS spectra, which permits redshifting of templates through simple pixel shifting. The trial redshifts corresponding to the five lowest $\chi^{2}$ values are then re-determined locally to subpixel accuracy, and errors in these values are determined from the curvature of the $\chi^{2}$ curve at the position of the minimum. 
Quasar redshifts are determined for all spectra in similar fashion to the galaxy redshifts, but over a larger range of exploration $(z=0.0333$ to $z=7.00)$ and with a larger initial velocity step $\left(276 \mathrm{~km} \mathrm{~s}^{-1}\right)$. The quasar eigenspectrum basis is defined by a PCA of 412 quasar spectra with known redshifts, and an underlying polynomial is allowed as well. Star redshifts are determined separately for each of 32 single subtype templates (excluding cataclysmic variables) using a single eigenspectrum plus a cubic polynomial for each sub-type, over a radial velocity range of $\pm 1200 \mathrm{~km} \mathrm{~s}^{-1}$. Only the single best radial velocity is retained for each stellar sub-type. Because of their intrinsic emission-line diversity, cataclysmic variable stars are handled differently from other stellar sub-types, with a three-component PCA eigenbasis plus quadratic polynomial, over a radial velocity range of $\pm 1000 \mathrm{~km} \mathrm{~s}^{-1}$. Visual inspection of thousands of galaxy, quasar, and cataclysmic variable star spectra (A. Bolton \& D. Schlegel 2011, private communication) demonstrate that the eigenspectra modeling is adequate, in the sense that the redshift error rate for spectra is of order $1 \%$, and the vast majority of the failures are flagged with a redshift warning flag (see the discussion in the DR6 paper).

Once the best five galaxy redshifts, best five quasar redshifts, and best stellar sub-type radial velocities for a given spectrum have been determined, these identifications are sorted in order of increasing reduced $\chi^{2}$, and the difference in reduced $\chi^{2}$ between each fit and the next-best fit with a radial velocity difference of greater than $1000 \mathrm{~km} \mathrm{~s}^{-1}$ is computed. The combination of redshift and template class that yields the lowest reduced $\chi^{2}$ is adopted as the pipeline measurement of the redshift and classification of the spectrum. Redshifts are corrected to the heliocentric frame. Several warning flags can be set (Table 4 of the DR6 paper) to indicate low confidence in this identification. The most common flag ("CHI2_CLOSE") is set to indicate that the change in reduced $\chi^{2}$ between the best and next-best redshift/classification is less than 0.01 .

Stellar redshifts are recomputed using the ELODIE library spectra as templates, after pruning to remove double and emission-line stars and anything else unsuitable for use as a velocity template. These redshifts represent our best estimate of the velocity of the star. Note, however, that the velocity errors are poorly characterized for the coolest (brown dwarf) and hottest (white dwarf) stars. See Schmidt et al. (2010) and West et al. (2011) for independent radial velocity measurements of SDSS L and $\mathrm{M}$ dwarfs, respectively.

As described in the DR6 paper, there is a systematic offset of $7.3 \mathrm{~km} \mathrm{~s}^{-1}$ in the stellar radial velocities measured with the ELODIE templates; this offset is corrected in the stellar parameters table in DR8. The rms plate-to-plate zero-point error in stellar velocities is $1.8 \mathrm{~km} \mathrm{~s}^{-1}$, as measured using the approximately 30 stars that are repeated on the bright and faint plates on each SEGUE-1 pointing. At $r=18$, about the median $\mathrm{S} / \mathrm{N}$ of the SEGUE stellar data, the total rms velocity error (including the contribution from the zero point) is about $4.4 \mathrm{~km} \mathrm{~s}^{-1}$, based on repeat observations.

At the best galaxy redshift, the stellar velocity dispersion is also determined by computing a PCA basis of eigenspectra from the ELODIE stellar library (Prugniel \& Soubiran 2001), convolved and binned to match the instrumental resolution and constant-velocity pixel scale of the reduced SDSS spectra, and broadened by Gaussian kernels of successively larger velocity width ranging from 0 to $850 \mathrm{~km} \mathrm{~s}^{-1}$ in steps of $25 \mathrm{~km} \mathrm{~s}^{-1}$. The broadened stellar template sets are redshifted to the best-fit galaxy redshift, and the spectrum is modeled as a least-squares linear combination of the basis at each trial broadening, masking pixels at the position of common emission lines in the galaxyredshift rest frame. The dependence of $\chi^{2}$ on assumed velocity dispersion allows a determination of the velocity dispersion and its error. The error is set to a negative value if the best value occurs at the high-velocity end of the fitting range. Reported best-fit velocity-dispersion values less than about $100 \mathrm{~km} \mathrm{~s}^{-1}$ are below the resolution limit of the SDSS spectrograph and are less reliable (see the discussion in the DR6 paper).

Flux values, redshifts, line widths, and continuum levels are computed for common rest-frame ultraviolet and optical emission lines by fitting multiple Gaussian-plus-background models at their observed positions within the spectra. The initial-estimate emission-line redshift is taken from the main redshift analysis, but is subsequently re-fit nonlinearly in the emission-line fitting routine. All lines are constrained to have the same redshift except for $\operatorname{Ly} \alpha$ (because of the bias induced by absorption from the $\operatorname{Ly} \alpha$ forest); note that this is not a perfect assumption for all quasar lines (e.g., Richards et al. 2002a; Shen et al. 2008). Intrinsic line widths are constrained to be the same for all emission lines, with the exception of the hydrogen Balmer series, which is given its own line width as a free parameter, and Ly $\alpha$ and NV $1241 \AA$, which each have their own free line-width parameters. Known 3:1 line flux ratios for the [O III] 4959, $5007 \AA$ and [N II] 6548, $6583 \AA$ doublets are imposed. When the $\mathrm{S} / \mathrm{N}$ of the line measurements permits doing so, spectra classified as galaxies are sub-classified into active galactic nucleus (AGN) and star-forming galaxies based upon measured $[\mathrm{O} \mathrm{III}] / \mathrm{H} \beta$ and $[\mathrm{N} \mathrm{II}] / \mathrm{H} \alpha$ line ratios (Baldwin et al. 1981, hereafter BPT), and galaxies with very high equivalent width in $\mathrm{H} \alpha$ are sub-classified as starburst objects. In the following section, we describe an alternative method to measure emission-line strengths.

\subsection{Quantities Derived from Galaxy Spectra}

\subsubsection{Galaxy Emission Lines}

In measuring the nebular emission lines of galaxies, it is important to properly account for the galaxy continuum which is very rich in stellar absorption features. The spectro1d pipeline (Subbarao et al. 2002) used in DR7 performs a simple estimate of the continuum using a sliding median. The idlspec $2 \mathrm{~d}$ code described in Section 4.2 uses a PCA technique to model the stellar continuum, which has the disadvantage that it is not constrained to produce astrophysically meaningful solutions. In DR8, we offer a third set of emission-line measurements for galaxy spectra, which makes use of stellar population synthesis models to accurately fit and subtract the stellar continuum. The code has been run on previous SDSS data releases and the resulting measurements used for a variety of scientific applications (e.g., Tremonti et al. 2004; Brinchmann et al. 2004; Kauffmann et al. 2003b). These data have been publicly available $^{80}$ since DR4; we are making them accessible through the SDSS data release for the first time with DR8. We refer to this set of line measurements as the MPA-JHU measurements, after the Max Planck Institute for Astrophysics and the Johns Hopkins University where the technique was developed. We provide MPA measurements for all objects that idlspec $2 \mathrm{~d}$ calls a galaxy; see Section 4.2. We briefly describe the technique here; details can be found in C. Tremonti et al. (2011, in preparation).

We first scale each galaxy spectrum to match its $r$-band fiber magnitude and correct each spectrum for Galactic extinction

\footnotetext{
80 http://www.mpa-garching.mpg.de/SDSS/
} 

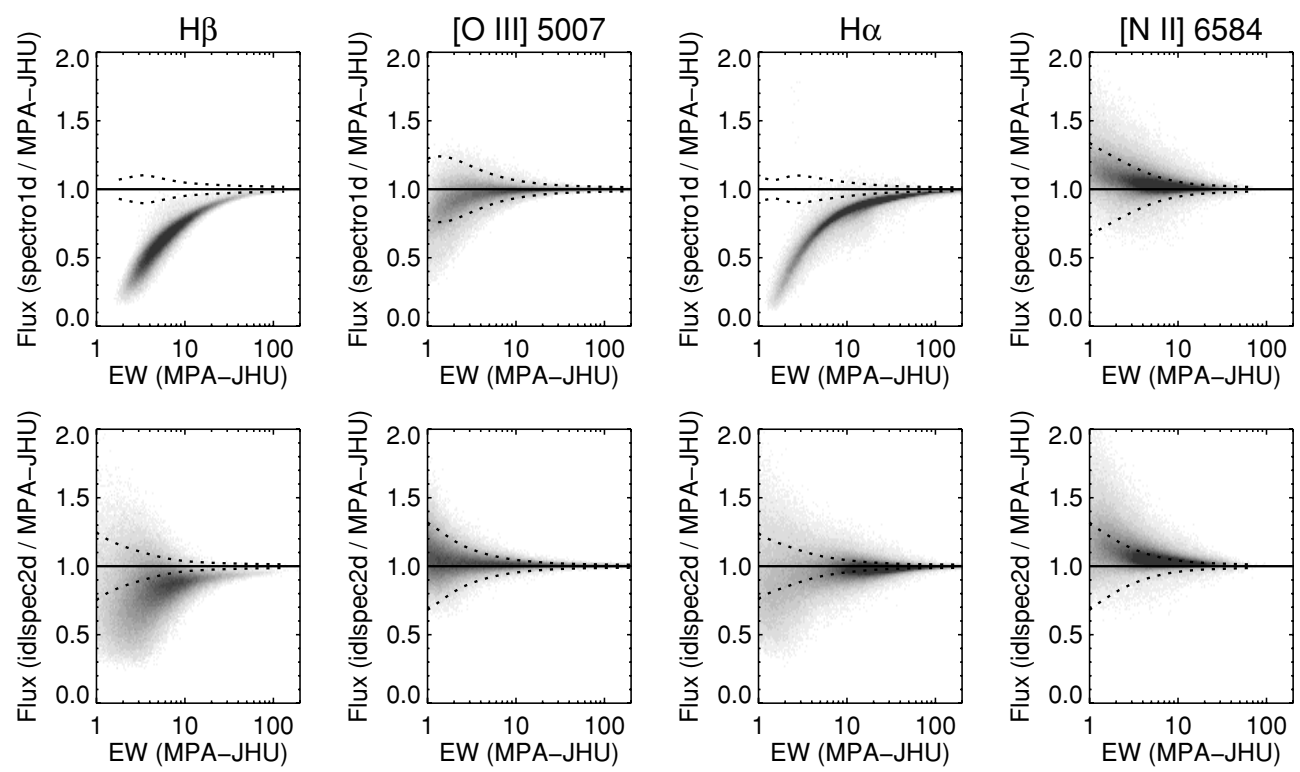

Figure 5. Ratio of the spectro1d and idlspec2d emission-line flux measurements with those of the MPA-JHU pipeline, as a function of rest-frame equivalent width, for galaxies in DR8 with emission-line measurements with greater than $3 \sigma$ significance. In performing this comparison, we have put all measurements on a common scale by removing the Milky Way reddening and spectrophotometric zero-point corrections from the MPA-JHU line measurements. The remaining differences are due to the different methods of modeling the stellar continuum. The dotted lines show the deviation expected due to random error.

following SFD and the O'Donnell (1994) attenuation curve. We adopt the basic assumption that any galaxy star formation history can be approximated as a sum of discrete bursts. Our library of template spectra is composed of single stellar population models generated using the population synthesis code of Bruzual \& Charlot (2003). We have used a new version kindly made available by the authors which incorporates the MILES empirical spectral library (Sánchez-Blázquez et al. 2006; these spectra cover the range 3525-7500 $\mathrm{A}$ with $2.3 \AA$ AWHM). The spectral-type and metallicity coverage, fluxcalibration accuracy, and number of stars in the library represent a substantial improvement over previous libraries. Our templates include models of 10 different ages $(0.005,0.025,0.1,0.2,0.6$, $0.9,1.4,2.5,5$, and $10 \mathrm{Gyr})$ and four metallicities $(1 / 4,1 / 2$, 1 , and $2.4 Z_{\odot}$ ). For each galaxy, we transform the templates to the measured redshift and velocity dispersion and resample them to match the data. To construct the best-fitting model, we perform a non-negative least-squares fit to a linear combination of our 10 single-age populations, with internal dust attenuation modeled as an additional free parameter following Charlot \& Fall (2000). Given the S/N of the spectra, we model galaxies as single metallicity populations and select the metallicity that yields the minimum $\chi^{2}$.

After subtracting the best-fitting stellar population model of the continuum, we remove any remaining residuals (usually of order a few percent) with a sliding 150 pixel median and fit all the nebular emission lines simultaneously as Gaussians. In doing so, we require that the Balmer lines $(\mathrm{H} \delta, \mathrm{H} \gamma, \mathrm{H} \beta$, and $\mathrm{H} \alpha)$ have the same line width and velocity offset, and likewise for the forbidden lines (e.g., [O II] $\lambda \lambda 3726,3729$, [O III] $\lambda \lambda 4959,5007$, [N II] $\lambda \lambda 6548,6584$, [S II] $\lambda \lambda 6717,6731)$. We take into account the wavelength-dependent instrumental resolution of each fiber, which is measured by the idlspec2d pipeline from the arc lamp images.

In Figure 5, we explore the differences in the line fluxes measured by the MPA-JHU, spectro1d and idlspec2d codes resulting from the differences in modeling the stellar continuum. The line fluxes of [O III] $\lambda 5007$ and $[\mathrm{N}$ II] $\lambda 6584$ are generally consistent within the errors. The Balmer lines are systematically underestimated by spectro1d at low equivalent widths because stellar Balmer absorption has not been accounted for by the smooth continuum model they used. The differences are smaller when comparing the MPA-JHU and idlspec $2 \mathrm{~d}$ measurements, since both codes model the stellar continuum in detail, but they are still significant for $\mathrm{H} \beta$.

The idlspec2d and MPA-JHU codes also show significant differences in equivalent width measurements of Balmer lines at high equivalent width. The idlspec2d code records the continuum at line center of the best-fit stellar continuum model (corresponding to the trough of Balmer stellar absorption lines), while the MPA-JHU code median smooths the emission-line-subtracted spectrum by 100 pixels $\left(\sim 6900 \mathrm{~km} \mathrm{~s}^{-1}\right)$ before recording the continuum at line center. For galaxies with significant intermediate age stellar populations, the differences between the two continuum measurements can be as large as $30 \%$, which has a correspondingly large effect on line equivalent widths.

\subsubsection{Physical Properties of Galaxies}

DR8 also includes a number of galaxy physical parameters derived by the MPA-JHU group.

1. BPT classification. We supply emission-line classifications based on the BPT diagram, [N II] 6584/H $\alpha$ versus [O III] 5007/H $\beta$. Galaxies are divided into Star Forming, Composite, AGN, Low S/N Star Forming, Low S/N AGN, and Unclassifiable categories as outlined in Brinchmann et al. (2004).

2. Stellar mass. Stellar masses are calculated using the Bayesian methodology and model grids described in Kauffmann et al. (2003a). The spectra are measured through a $3^{\prime \prime}$ aperture and therefore do not represent the entire galaxy. We therefore base our model on the ugriz galaxy photometry alone (rather than the spectral indices $\mathrm{D}_{n}(4000)$ and $\mathrm{H} \delta_{A}$ used by Kauffmann et al. 2003a). We have corrected the photometry for the small contribution due to 
nebular emission using the spectra. We estimate the stellar mass within the SDSS spectroscopic fiber aperture using fiber magnitudes and the total stellar mass using model magnitudes. A Kroupa (2001) initial mass function is assumed. We output the stellar mass corresponding to the median and $2.5 \%, 16 \%, 84 \%$, and $97.5 \%$ of the probability distribution function.

3. Nebular oxygen abundance. Nebular oxygen abundances are estimated from the strong optical emission lines ([O II] 3727, H $\beta$, [O III] 5007, [N II] 6548, 6584, and [S II] 6717, 6731) using the Bayesian methodology outlined in Tremonti et al. (2004) and Brinchmann et al. (2004). Oxygen abundances are only computed for objects classified as Star Forming. We output the value of $12+\log (\mathrm{O} / \mathrm{H})$ at the median and $2.5 \%, 16 \%, 84 \%$, and $97.5 \%$ of the probability distribution function.

4. Star formation rate. Star formation rates (SFRs) are computed within the galaxy fiber aperture using the nebular emission lines as described in Brinchmann et al. (2004). SFRs outside of the fiber are estimated using from fits of model grids to the $u, g, r, i, z$ photometry outside the fiber, following the method described in Salim et al. (2007). ${ }^{81}$ The same technique was also applied to estimate SFRs in AGN and galaxies with weak emission lines. We report both the fiber SFR and the total SFR at the median and 2.5\%, 16\%, $84 \%$, and $97.5 \%$ of the probability distribution function.

5. Specific SFR. The specific SFR (the ratio SFR to the stellar mass) has been calculated by combining the SFR and stellar mass likelihood distributions as outlined in Appendix A of Brinchmann et al. (2004). We report both the fiber and the total specific SFR at the median and $2.5 \%, 16 \%, 84 \%$, and $97.5 \%$ of the probability distribution function.

\subsection{Changes to SSPP}

The SSPP (Lee et al. 2008a, 2008b; Allende Prieto et al. 2008) fits models to SDSS spectra of stars in order to determine surface temperature, gravity, and metallicity. The pipeline was refined for SEGUE-2 to improve the parameter estimates, as described in the Appendix of Smolinski et al. (2011). This refined version, which we summarize here, has been used for the DR8 processing.

The SSPP uses multiple techniques to estimate $[\mathrm{Fe} / \mathrm{H}]$, effective temperature, and surface gravity. Each of these methods is considered valid over a particular range of $g-r$ and $\mathrm{S} / \mathrm{N}$, and some methods are more accurate or better calibrated at low or high metallicity. To choose between them, we compare the observed and model spectra at the metallicity given by each method and reject those for which the correlation coefficient between the spectra or the mean residuals are poor. This approach has improved the accuracy of metallicity estimates for stars up to solar metallicity, as demonstrated in particular by the SSPP parameters for stars in M67 in Smolinski et al. (2011). Further work on reducing bias in the SSPP in other parts of the $\mathrm{H}-\mathrm{R}$ diagram came from adjusting the $g-r$ and $\mathrm{S} / \mathrm{N}$ ranges for some estimators, and recalibration of others using the cluster plates (Section 4.6) and high-resolution data taken on other telescopes. The SSPP reports stellar parameters for stars in the range $-0.3<g-r<1.3$, but below $g-r=0.0\left(T_{\text {eff }}=\right.$ $7500 \mathrm{~K})$ or above $g-r=0.8\left(T_{\text {eff }}=4500 \mathrm{~K}\right)$, the errors in $T_{\text {eff }}$ and $\log g$ become appreciably larger.

\footnotetext{
81 The SFRs provided on the MPA-JHU Web site use a slightly different technique for galaxies for weak emission lines, as will be described in J. Brinchmann et al. (2011, in preparation).
}

The SSPP now also includes estimates of metallicity, gravity, and temperature based on the spectra alone, with no photometric information. These "spectroscopic only" parameter estimates are more reliable in regions of high extinction (J. Cheng et al. 2011, in preparation). Finally, the SSPP reports metallicity and gravity estimates made with the effective temperature determined from a color-temperature relation; these may provide more reliable parameter estimates for low-metallicity stars.

\subsection{Spectroscopic Data Quality}

Each spectroscopic plate is assigned a quality (PLATEQUALITY) with one of three values: "good," a good science quality plate; "marginal," an acceptable plate, but lower quality than good plates; and "bad," a plate with results that should be treated with skepticism.

The PLATEQUALITY value is set independently for each observation (labeled by the MJD of the observation) of each plate. For Legacy plates, the definition of plate quality is based on the median squared $\mathrm{S} / \mathrm{N}$ per spectroscopic pixel for targets at $g_{\text {fiber }}=20\left((\mathrm{~S} / \mathrm{N})^{2}\right.$ in what follows $)$ and the fraction $f_{\mathrm{bad}}$ of pixels in the sky fibers that have $\chi^{2}>4$ in the model for the sky spectrum in any of the contributing exposures. In particular, a plate with $(\mathrm{S} / \mathrm{N})^{2}>15$ and $f_{\text {bad }}<0.05$ is deemed "good;" a plate with $(\mathrm{S} / \mathrm{N})^{2}>9$ and $f_{\text {bad }}<0.13$ is deemed "marginal;" and otherwise it is deemed "bad."

For SEGUE plates, the conditions are based on the S/N of main-sequence turnoff stars at $g=18$. For faint SEGUE-1 plates, a plate with $(\mathrm{S} / \mathrm{N})^{2}>16$ is deemed "good." For bright SEGUE-1 plates, a plate with $(\mathrm{S} / \mathrm{N})^{2}>7.5$ is deemed "good." SEGUE-2 plates with $(\mathrm{S} / \mathrm{N})^{2}>10$ are considered "good." SEGUE-1 and SEGUE-2 plates do not have a "marginal" quality designation. Finally, for plates observed during the first stages of commissioning, low Galactic latitude plates, and cluster plates (Section 4.6), the quality is set by visual inspection of the data.

Three additional flags provide more detail on the nature of the plate. IS_BEST is set to 1 if a given observation is the best observation of a plate (whether or not it is marked as bad), and 0 otherwise. IS_PRIMARY is set to 1 if the plate is the best observation of a given plate (i.e., IS_BEST is set), and the observation is not marked as "bad," and 0 otherwise. Finally, IS_TILE is set to 1 if the plate is the best Legacy plate covering its location, and 0 otherwise; the definition of the Legacy spectroscopy is the union of all plates with IS_TILE set. A plate can only be IS_TILE if it is also IS_PRIMARY.

Selecting plates which are not "bad" will yield a good sample of spectra. Nevertheless, many of the "bad" plates actually contain useful data (in particular, many highly reliable redshifts). However, bad plates should be treated with care (in particular, they may have poor spectrophotometry or residual sky-subtraction problems).

\subsection{New and Reprocessed Plates}

In DR7 and previous data releases, there were a number of observations of plates that had been observed and reduced, but not included in the releases because they were of lower quality and/or were repeats of other plates. In DR8, we are releasing 108 such plates, with improved quality flags so that marginal or bad plates can be flagged in analysis. Twelve of these 108 plates are new, in the sense that they are not simply repeats of observations already included in DR7. Of these 108 plates, 24 are classified "good." 
SEGUE observed stars in a number of well-studied open and globular clusters, including M92, NGC5053, M53, M15, M13, M2, M3, NGC 2420, M67, NGC 6791, M71, Be 29, M35, NGC 2158, and NGC 7789 (C. Rockosi et al. 2011, in preparation; Smolinski et al. 2011; Z. Ma et al. 2011, in preparation). These clusters have well-measured metallicities and allow us to sample regions of the H-R diagram that we do not otherwise probe in the SDSS, so observations of these clusters are invaluable for calibrating the outputs of the SSPP. These so-called cluster plates were made available in DR7, but we faced some challenges in reducing them. Difficulties included background contamination in the target, flux calibration, and sky fibers due to the crowded fields, the lack of good-quality reductions of the relevant SDSS photometric data (indeed, we did not have SDSS imaging data at all for some clusters), and the large range of brightnesses of targets on a single plate, giving rise to cross-talk between adjacent fibers. For DR8, the cluster plates were reprocessed using careful iterative selection of the sky and flux-standard fibers. The required changes in the reduction procedure were small enough that the goal of having uniform reductions for the cluster calibration stars and the survey plates was met.

Because of the difficulty in finding good photometric standards for the reductions of the cluster plates, there are some lowlevel, large-scale residuals in the spectrophotometric solution. These residuals are corrected in the continuum normalization procedure in the SSPP, and the SSPP parameters are unaffected. However, users of these spectra should be aware of these and other possible systematic errors in the flux calibration.

\subsection{Matching Photometry to Spectroscopy}

In DR8, we introduce a new method for matching the photometry to the spectroscopy. Instead of a purely positional match that searches for the nearest photometric object center to a spectrum, we search for the object that, according to the photometric reductions, contributes the greatest amount of light to the spectrum. In detail, we quantify the contribution of light using a 3 " diameter aperture in the $r$ band. While this "fluxbased" match is the default that we provide in the data release, the "position-based" match is also provided. We do not correct for proper motion of stars between the time that the images and the spectra were taken.

The "flux-based" match is usually appropriate and typically more accurate for large, nearby galaxies. In particular, the latest photometric pipeline version often deblends parent objects into children differently than the version that was used for targeting. Therefore, the spectrum of a galaxy might be significantly offset from the location that we now deem to be its "center." The "flux-based" matches recover many such cases. The "position-based" match is important for other purposes such as spectrophotometry.

In more detail, we first execute a purely positional match to the primary photometric catalog for each spectrum, using a $2^{\prime \prime}$ matching criterion. For each spectrum, the matching photometric object id is stored in the field ORIGOBJID in the files and in the database. For the $\sim 1 \%$ of spectra that have no position-based match, we find the primary imaging field that contains the location of the spectrum. If there are no detected pixels at the location of the spectrum (that is, if it is not contained in a "parent" object), then the object is unmatched. This happens for about $90 \%$ of the objects without a position-based match; these objects are typically sky fibers or transient objects such as satellites, in cases where the primary imaging field in the final photometric catalog differs from the original field used to target the spectroscopy.

Some spectra with no position-based match nevertheless fall within the boundaries of some "parent" object. In these cases, we perform $3^{\prime \prime}$ diameter aperture photometry in the $r$ band at the location of the spectrum, using the atlas images of the parent and all of its children. The flux-based match is designated to be the child that contributes the most flux to the parent, and we store its object id as the BESTOBJID associated with the spectrum.

Finally, for spectra with a position-based match, we compare the $3^{\prime \prime}$ fiber flux with a $3^{\prime \prime}$ aperture flux based on the radial profile measured by photo. The fiber magnitude is based on the parent atlas image, whereas the radial profile is calculated using only the child atlas image. Therefore, in cases where our aperture flux is less than $50 \%$ of the fiber flux, the light in the fiber is dominated by other objects. In those cases, we perform aperture photometry at the fiber location on the atlas images of the parent and all children. We select the child with the most flux as the flux-based match and store its object id as the BESTOBJID associated with the spectrum.

About $0.5 \%$ of all spectra have flux-based matches that differ from the position-based matches. Typically, half of these are cases where the photometry is irretrievably bad in some way (such as the presence of a long satellite trail or airplane). The other half are cases where the flux-based match appears more appropriate when one examines the images by eye; that is, where the redshift of the spectrum should be associated with the fluxbased match in the photometric catalog.

\section{DATA DISTRIBUTION}

In SDSS-I/II, the data were distributed with two different portals. The CAS is a database containing catalogs of SDSS objects (both photometric and astrometric) that allowed queries on their measured attributes. The Data Archive Server (DAS) consists of flat files containing the images themselves, the catalogs, the spectra, and other data products. We continue to use the CAS for DR8; ${ }^{82}$ it is largely unchanged, although some obsolete tables and schema have been removed.

The design of the DR7 CAS considered the SDSS Legacy survey to be fundamental. Thus imaging objects that fell outside the Legacy footprint were flagged as secondary. The DR8 CAS does not keep this distinction; it treats all imaging runs as equivalent and uses the uniform results from the resolve algorithm (Section 3.4) across the entire unique imaging area.

The DAS functionality has been replaced with the SDSS-III Science Archive Server (SAS), ${ }^{83}$ which has a similar, but not identical, directory structure. In SDSS-I/II, the names of various fields and attributes differed between the DAS and CAS. More importantly, there was not a perfect match between the contents of the two: for example, there were imaging runs and spectroscopic plates available in the DAS that were not present in the CAS. We have endeavored to couple the CAS and the SAS more closely in DR8. To a very good approximation, the data contained in the two are the same, though packaged differently. In particular, unlike DR7, all the normal imaging scans included in the SAS are in the CAS as well.

In the DR7 DAS, the photometrically and astrometrically calibrated versions of these files were called tsObj or $\mathrm{drObj}$ files; the nomenclature of the uncalibrated and corresponding calibrated quantities was not always consistent (for example,

\footnotetext{
82 http://skyserver.sdss3.org/dr8/

$83 \mathrm{http}: / /$ data.sdss3.org
} 
some calibrated quantities had names, like psfCounts, that erroneously implied that they were not calibrated). This situation has been rectified in the so-called photoObj files found in the SAS and in the tables in the CAS. Similarly, the metadata files describing each field, which in DR7 were called tsField files, have a changed format, called photoField files, which includes information about the ubercalibration. The full data model with a definition of all terms may be found on the DR8 Web site.

In addition to the photoObj files, we also provide a much more compact version of the catalog called the "datasweeps" in the calibObj files. These files mirror the photoObj files but only list the most commonly used attributes for each object and only retain objects with a reasonable detection ${ }^{84}$ in at least one band. The datasweeps are convenient for users who need basic information for all objects in a compact form.

In DR7, only calibrated asinh magnitudes (Lupton et al. 1999) were tabulated, with names like psfMag. With DR8, we also include, for all photometric quantities, the linear flux density (i.e., no logarithms or asinh), in units of "nanomaggies" (Finkbeiner et al. 2004), with names like psfFlux. A nanomaggie (nMgy) is defined as the flux density (per unit frequency) of a $22.5 \mathrm{AB}$ magnitude object in any band. Given the definition of AB magnitudes (Oke \& Gunn 1983),

$1 \mathrm{nMgy}=3.631 \times 10^{-6} \mathrm{Jy}=3.631 \times 10^{-29} \mathrm{erg} \mathrm{s}^{-1} \mathrm{~cm}^{-2} \mathrm{~Hz}^{-1}$.

As in DR7, SAS makes available corrected frames of each field, in which defects have been interpolated over. However, unlike DR7, the DR8 versions of these files contain flux values calibrated in nanomaggies, have a global best-fit sky model (Section 3.2) subtracted, and have a proper WCS header. The calibration and sky-subtraction information is bundled with the files and can be easily backed out if necessary.

Finally, the SAS user interface is quite different from that of the DR7 DAS. In addition to allowing for searches for spectra based on coordinates, redshifts, target flags, and fiber identification numbers, it provides an interactive interface to plot the spectra. It also allows coordinate searches for fields, as well as returning FITS mosaics that stitch together overlapping fields.

\section{CONCLUSIONS}

This paper describes the eighth data release of the SDSS, consisting of all the SDSS data taken through Summer 2009, together with the final imaging of the southern Galactic cap completed in 2010 January. The images cover a footprint of over $14,500 \mathrm{deg}^{2}$; including repeat observations, the total quantity of imaging data is more than twice this value. All these data have been reprocessed with an updated version of the photometric pipeline, which gives modest improvements to the photometry of bright galaxies and fainter galaxies near them. In addition, DR8 contains the spectra of over 1.6 million galaxies, quasars, and stars, including 118,000 new stellar spectra from the SEGUE-2 survey, as well as 108 plates of data not previously released.

With the completion of the imaging survey, the SDSS camera has been retired. SDSS-III is described in detail in Eisenstein et al. (2011); it will continue through 2014. This release contains

\footnotetext{
84 Defined to be those stars for which the PSF magnitude in at least one of ( $u, g, r, i, z)$ is brighter than $(22.5,22.5,22.5,22,21.5)$, and those galaxies for which one model magnitude is brighter than $(21,22,22,20.5,20.1)$, after correction for Galactic extinction following SFD. This criterion excludes roughly $23 \%$ of the objects.
}

data from two of its four surveys: SEGUE-2 and the imaging component of BOSS. BOSS spectroscopy has started, and its first year of data will be made available as part of the ninth data release. Plots showing the quality of those data may be found in Eisenstein et al. (2011) and White et al. (2011). In addition, the MARVELS survey is well underway, and the first scientific results have been published (Lee et al. 2011). Finally, APOGEE will probably have seen first light by the time this paper is published, and data from that survey will first be released publicly in the tenth data release.

We thank the referee, Andrew West, for comments that improved the paper. Funding for SDSS-III has been provided by the Alfred P. Sloan Foundation, the Participating Institutions, the National Science Foundation, and the US Department of Energy. The SDSS-III Web site is http://www.sdss3.org/.

SDSS-III is managed by the Astrophysical Research Consortium for the Participating Institutions of the SDSS-III Collaboration including the University of Arizona, the Brazilian Participation Group, Brookhaven National Laboratory, University of Cambridge, University of Florida, the French Participation Group, the German Participation Group, the Instituto de Astrofisica de Canarias, the Michigan State/Notre Dame/JINA Participation Group, Johns Hopkins University, Lawrence Berkeley National Laboratory, Max Planck Institute for Astrophysics, New Mexico State University, New York University, Ohio State University, Pennsylvania State University, University of Portsmouth, Princeton University, the Spanish Participation Group, University of Tokyo, University of Utah, Vanderbilt University, University of Virginia, University of Washington, and Yale University.

\section{REFERENCES}

Abazajian, K., et al. 2003, AJ, 126, 2018 (DR1 Paper)

Abazajian, K., et al. 2004, AJ, 128, 502 (DR2 Paper)

Abazajian, K., et al. 2005, AJ, 129, 1755 (DR3 Paper)

Abazajian, K., et al. 2009, ApJS, 182, 543 (DR7 Paper)

Adelman-McCarthy, J. K., et al. 2006, ApJS, 162, 38 (DR4 Paper)

Adelman-McCarthy, J. K., et al. 2007, ApJS, 172, 634 (DR5 Paper)

Adelman-McCarthy, J. K., et al. 2008, ApJS, 175, 297 (DR6 Paper)

Allende Prieto, C., et al. 2008, AJ, 136, 2070

An, D., et al. 2008, ApJS, 179, 326

Baldwin, J. A., Phillips, M. M., \& Terlevich, R. 1981, PASP, 93, 5 (BPT)

Bernardi, M., et al. 2007, AJ, 133, 1954

Berriman, G. B., Good, J. C., Curkendall, D. W., Jacob, J. C., Katz, D. S., Prince, T. A., \& Williams, R. 2003, in ASP Conf. Ser. 295, Astronomical Data Analysis Software and Systems XII, ed. H. E. Payne, R. I. Jedrzejewski, \& R. N. Hook (San Francisco, CA: ASP), 343

Blanton, M. R., Kazin, E., Muna, D., Weaver, B. A., \& Price-Whelan, A. 2011, AJ, submitted

Blanton, M. R., et al. 2005, ApJ, 631, 208

Brinchmann, J., Charlot, S., White, S. D. M., Tremonti, C., Kauffmann, G., Heckman, T., \& Brinkmann, J. 2004, MNRAS, 351, 1151

Bruzual, G., \& Charlot, S. 2003, MNRAS, 344, 1000

Charlot, S., \& Fall, S. M. 2000, ApJ, 539, 718

Clem, J. L., Vanden Berg, D. A., \& Stetson, P. B. 2008, AJ, 135, 682

Collinge, M., et al. 2005, AJ, 129, 2542

Dufour, P., et al. 2007, ApJ, 663, 1291

Eisenstein, D. J., et al. 2001, AJ, 122, 2267

Eisenstein, D. J., et al. 2011, AJ, submitted (arXiv:1101.1529)

Feldmann, R., et al. 2006, MNRAS, 372, 565

Finkbeiner, D. P., et al. 2004, AJ, 128, 2577

Frieman, J. A., et al. 2008, AJ, 135, 338

Fukugita, M., Ichikawa, T., Gunn, J. E., Doi, M., Shimasaku, K., \& Schneider, D. P. 1996, AJ, 111, 1748

Fukugita, M., et al. 2007, AJ, 134, 579

Glazebrook, K., Offer, A. R., \& Deeley, K. 1998, ApJ, 492, 98

Gunn, J. E., et al. 1998, AJ, 116, 3040

Gunn, J. E., et al. 2006, AJ, 131, 2332 
Hall, P. B., et al. 2002, ApJS, 141, 267

Hogg, D. W., Finkbeiner, D. P., Schlegel, D. J., \& Gunn, J. E. 2001, AJ, 122, 2129

Ivezić, Ž., et al. 2004, Astron. Nachr., 325, 583

Kaiser, N., et al. 2002, Proc. SPIE, 4836, 154

Kauffmann, G., et al. 2003a, MNRAS, 341, 33

Kauffmann, G., et al. 2003b, MNRAS, 346, 1055

Kessler, R., et al. 2009, ApJS, 185, 32

Kollmeier, J. A., et al. 2010, ApJ, 723, 812

Kroupa, P. 2001, MNRAS, 322, 231

Lauer, T. R., et al. 2007, ApJ, 662, 808

Lee, B. L., et al. 2011, ApJ, 728, 32

Lee, Y. S., et al. 2008a, AJ, 136, 2022

Lee, Y. S., et al. 2008b, AJ, 136, 2050

Lenz, D. D., Newberg, H. J., Rosner, R., Richards, G. T., \& Stoughton, C. 1998, ApJS, 119, 121

Lépine, S., \& Scholz, R.-D. 2008, ApJ, 681, L33

Lintott, C., et al. 2008, MNRAS, 389, 1179

Lintott, C., et al. 2011, MNRAS, 410, 166

Lisker, T., Glatt, K., Westera, P., \& Grebel, E. K. 2006, AJ, 132, 2432

Lupton, R. H., Gunn, J. E., Ivezić, Ž., Knapp, G. R., Kent, S., \& Yasuda, N. 2001, in ASP Conf. Ser. 238, Astronomical Data Analysis Software and Systems X, ed. F. R. Harnden, Jr., F. A. Primini, \& H. E. Payne (San Francisco, CA: ASP), 269

Lupton, R. H., Gunn, J. E., \& Szalay, A. S. 1999, AJ, 118, 1406

Mandelbaum, R., et al. 2005, MNRAS, 361, 1287

Ménard, B., Scranton, R., Fukugita, M., \& Richards, G. T. 2010, MNRAS, 405, 1025

Monet, D. G., et al. 2003, AJ, 125, 984

Munn, J. A., et al. 2004, AJ, 127, 3034

O’Donnell, J. E. 1994, ApJ, 422, 158

Oke, J. B., \& Gunn, J. E. 1983, ApJ, 266, 713

Padmanabhan, N., et al. 2008, ApJ, 674, 1217

Petrosian, V. 1976, ApJ, 209, L1

Pier, J. R., Munn, J. A., Hindsley, R. B., Hennessy, G. S., Kent, S. M., Lupton, R. H., \& Ivezić, Ž. 2003, AJ, 125, 1559
Plotkin, R. M., et al. 2010, AJ, 139, 390

Prugniel, P., \& Soubiran, C. 2001, A\&A, 369, 1048

Richards, G. T., Vanden Berk, D. E., Reichard, T. A., Hall, P. B., Schneider,

D. P., SubbaRao, M., Thakar, A. R., \& York, D. G. 2002a, AJ, 124, 1

Richards, G. T., et al. 2002b, AJ, 123, 2945

Salim, S., et al. 2007, ApJS, 173, 267

Sánchez-Blázquez, P., et al. 2006, MNRAS, 371, 703

Schlafly, E. F., Finkbeiner, D. P., Schlegel, D. J., Jurić, M., Ivezić, Ž., Gibson, R. R., Knapp, G. R., \& Weaver, B. A. 2010, ApJ, 725, 1175

Schlegel, D. J., Finkbeiner, D. P., \& Davis, M. 1998, ApJ, 500, 525 (SFD)

Schmidt, S. J., West, A. A., Hawley, S. L., \& Pineda, J. S. 2010, AJ, 139, 1808

Schmidt, G. D., et al. 2003, ApJ, 595, 1101

Schneider, D. P., et al. 2010, AJ, 139, 2360

Shen, Y., Greene, J. E., Strauss, M. A., Richard, G. T., \& Schneider, D. P. 2008, ApJ, 680, 169

Skrutskie, M. F., et al. 2006, AJ, 131, 1163

Smith, J. A., et al. 2002, AJ, 123, 2121

Smolinski, J. P., et al. 2011, AJ, 141, 89

Stoughton, C., et al. 2002, AJ, 123, 485 (EDR Paper)

Strauss, M. A., et al. 2002, AJ, 124, 1810

Subbarao, M., Frieman, J., Bernardi, M., Loveday, J., Nichol, B., Castander, F., \& Meiksin, A. 2002, Proc. SPIE, 4847, 452

Swanson, M. E. C., Tegmark, M., Hamilton, A. J. S., \& Hill, C. J. 2008, MNRAS, 387,1391

Tremonti, C. A., et al. 2004, ApJ, 613, 898

Tucker, D., et al. 2006, Astron. Nachr., 327, 821

West, A. A., Garcia-Appadoo, D. A., Dalcanton, J. J., Disney, M. J., Rockosi, C. M., Ivezić, Z., Bentz, M. C., \& Brinkmann, J. 2010, AJ, 139, 315

West, A. A., et al. 2011, AJ, 141, 97

White, M., et al. 2011, ApJ, 728, 126

Yanny, B., et al. 2009, AJ, 137, 4377

York, D. G., et al. 2000, AJ, 120, 1579

Zacharias, N., Urban, S. E., Zacharias, M. I., Wycoff, G. L., Hall, D. M., Monet, D. G., \& Raffert, T. J. 2004, AJ, 127, 3043 


\section{ERRATUM: “THE EIGHTH DATA RELEASE OF THE SLOAN DIGITAL SKY SURVEY: FIRST DATA FROM SDSS-III” (2011, ApJS, 193, 29)}

Hiroaki Aihara ${ }^{1}$, Carlos Allende Prieto $^{2,3}$, Deokkeun An ${ }^{4}$, Scott F. Anderson ${ }^{5}$, Éric Aubourg ${ }^{6,7}$, Eduardo Balbinot ${ }^{8,9}$, Timothy C. Beers ${ }^{10}$, Andreas A. Berlind ${ }^{11}$, Steven J. Bickerton ${ }^{12}$, Dmitry Bizyaev ${ }^{13}$, Michael R. Blanton ${ }^{14}$, John J. Bochanski ${ }^{15}$, Adam S. Bolton ${ }^{16}$, Jo Bovy ${ }^{14}$, W. N. Brandt ${ }^{15}, 17$, J. Brinkmann ${ }^{13}$, Peter J. Brown ${ }^{16}$, Joel R. Brownstein ${ }^{16}$, Nicolas G. Busca ${ }^{6}$, Heather Campbell ${ }^{18}$, Michael A. Carr ${ }^{12}$, Yanmei Chen ${ }^{19}$, Cristina Chiappini ${ }^{9}, 20,21$, Johan Comparat ${ }^{22}$, Natalia Connolly ${ }^{23}$, Marina Cortes ${ }^{24}$, Rupert A. C. Croft ${ }^{25}$, Antonio J. Cuesta ${ }^{26}$, Luiz N. da Costa ${ }^{9,27}$, James R. A. DavenPort ${ }^{5}$, Kyle Dawson $^{16}$, Saurav Dhital ${ }^{11}$, Anne Ealet ${ }^{28}$, Garrett L. Ebelke ${ }^{13,29}$, Edward M. Edmondson ${ }^{18}$, Daniel J. Eisenstein ${ }^{30,31}{ }^{3}$ STePhanie Escoffier $^{28}$, Massimiliano Esposito ${ }^{2,3}$, Michael L. Evans ${ }^{5}$, Xiaohui Fan ${ }^{30}$, Bruno Femenía Castella ${ }^{2,3}$, Andreu Font-Ribera ${ }^{32}$,

Peter M. Frinchaboy ${ }^{33}$, Jian Ge ${ }^{34}$, Bruce A. Gillespie ${ }^{13}$, G. Gilmore ${ }^{35}$, Jonay I. GonZález Hernández ${ }^{2}$, J. Richard GotT ${ }^{12}$, Andrew Gould ${ }^{36}$, Eva K. Grebel ${ }^{37}$, James E. GunN ${ }^{12}$, Jean-Christophe Hamilton ${ }^{6}$, Paul Harding ${ }^{38}$, David W. Harris ${ }^{16}$, SuZanne L. Hawley ${ }^{5}$, Frederick R. Hearty ${ }^{39}$, Shirley Ho ${ }^{24}$, David W. Hogg ${ }^{14}$, Jon A. Holtzman ${ }^{29}$,

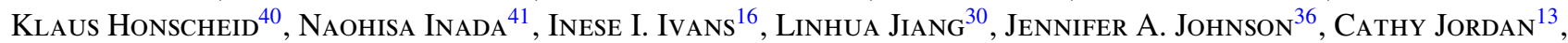
Wendell P. Jordan ${ }^{13,29}$, Eyal A. Kazin ${ }^{14}$, David KirkbY ${ }^{42}$, Mark A. Klaene ${ }^{13}$, G. R. Knapp ${ }^{12}$, Jean-Paul Kneib ${ }^{22}$, C. S. KochaneK ${ }^{36}$, Lars Koesterke ${ }^{43}$, Juna A. Kollmeier ${ }^{44}$, Richard G. Kron ${ }^{45,46}$, Hubert Lampeitl ${ }^{18}$, Dustin Lang $^{12}$, Jean-Marc Le GofF ${ }^{7}$, Young Sun LeE ${ }^{10}$, Yen-Ting Lin ${ }^{1,47}$, Daniel C. Long ${ }^{13}$, Craig P. Loomis ${ }^{12}$, Sara Lucatello ${ }^{48}$,

Britt Lundgren ${ }^{26}$, Robert H. Lupton ${ }^{12}$, Zhibo Ma ${ }^{38}$, Nicholas MacDonald ${ }^{5}$, Suvrath Mahadevan ${ }^{15,49}$,

Marcio A. G. Maia ${ }^{9,27}$, Martin Makler ${ }^{9,50}$, Elena Malanushenko ${ }^{13}$, Viktor Malanushenko ${ }^{13}$, Rachel Mandelbaum ${ }^{12}$, Claudia Maraston ${ }^{18}$, Daniel Margala ${ }^{42}$, Karen L. Masters ${ }^{18}$, Cameron K. McBride $^{11}$, Peregrine M. McGehee ${ }^{51}$, Ian D. McGreer ${ }^{30}$, Brice Ménard ${ }^{1,52,53}$, Jordi Miralda-Escudé ${ }^{54,55}$, Heather L. Morrison $^{38}$, F. Mullally ${ }^{12,56}$, Demitri Muna ${ }^{14}$, Jeffrey A. Munn ${ }^{57}$, Hitoshi Murayama ${ }^{1}$, Adam D. Myers ${ }^{58}$, Tracy Naugle ${ }^{13}$, Angelo Fausti Neto ${ }^{8,9}$, Duy Cuong NGuYen ${ }^{34}$, Robert C. Nichol ${ }^{18}$, Robert W. O'CONNell ${ }^{39}$, Ricardo L. C. Ogando ${ }^{9,27}$,

Matthew D. Olmstead ${ }^{16}$, Daniel J. Oravetz ${ }^{13}$, Nikhil Padmanabhan ${ }^{26}$, Nathalie Palanque-Delabrouille ${ }^{7}$, Kaike Pan ${ }^{13}$, Parul Pandey ${ }^{16}$, Isabelle Pâris ${ }^{59}$, Will J. Percival ${ }^{18}$, Patrick Petitjean ${ }^{59}$, Robert Pfaffenberger ${ }^{29}$, Janine Pforr $^{18}$, Stefanie Phleps ${ }^{60}$, Christophe Pichon ${ }^{59}$, Matthew M. Pieri ${ }^{36,61}$, Francisco Prada ${ }^{62}$,

Adrian M. Price-Whelan ${ }^{14}$, M. Jordan RaddicK ${ }^{53}$, Beatriz H. F. Ramos ${ }^{9,27}$, CÉline Reylé63 ${ }^{63}$ James Rich $^{7}$, Gordon T. Richards ${ }^{64}$, Hans-Walter Rix ${ }^{65}$, Annie C. Robin ${ }^{63}$, Helio J. Rocha-Pinto ${ }^{9,66}$, Constance M. Rockosi ${ }^{67}$,

Natalie A. Roe ${ }^{24}$, Emmanuel Rollinde ${ }^{59}$, Ashley J. Ross ${ }^{18}$, Nicholas P. Ross ${ }^{24}$, Bruno M. Rossetto ${ }^{9,66}$,

Ariel G. Sánchez ${ }^{60}$, Conor Sayres ${ }^{5}$, David J. Schlegel ${ }^{24}$, Katharine J. Schlesinger ${ }^{36}$, Sarah J. SchmidT ${ }^{5}$,

Donald P. Schneider ${ }^{15,49}$, Erin Sheldon ${ }^{68}$, Yiping Shu ${ }^{16}$, Jennifer Simmerer ${ }^{16}$, Audrey E. Simmons ${ }^{13}$,

Thirupathi Sivarani ${ }^{34,69}$, STephanie A. Snedden ${ }^{13}$, Jennifer S. Sobeck ${ }^{46}$, Matthias Steinmetz $^{20}$, Michael A. Strauss ${ }^{12}$, Alexander S. Szalay ${ }^{53}$, Masayuki Tanaka ${ }^{1}$, Aniruddha R. Thakar ${ }^{53}$, Daniel Thomas ${ }^{18}$, Jeremy L. Tinker ${ }^{14}$, Benjamin M. Tofflemire ${ }^{5}$, Rita Tojeiro ${ }^{18}$, Christy A. Tremonti ${ }^{19}{ }^{2}$ Jan Vandenberg $^{53}$, M. Vargas Magaña ${ }^{6}$, Licia Verde ${ }^{54,55}$, Nicole P. Vogt ${ }^{29}$, David A. Wake ${ }^{26}$, Ji Wang ${ }^{34}$, Benjamin A. Weaver ${ }^{14}$, David H. Weinberg ${ }^{36}$, Martin White $^{70}$, Simon D. M. White ${ }^{71}$, Brian YannY ${ }^{45}$, NaOKi Yasuda ${ }^{1}$, Christophe YeChe $^{7}$, and Idit Zehavi ${ }^{38}$

${ }^{1}$ Institute for the Physics and Mathematics of the Universe, The University of Tokyo, Kashiwa 277-8583, Japan

${ }^{2}$ Instituto de Astrofísica de Canarias, E38205 La Laguna, Tenerife, Spain

${ }^{3}$ Departamento de Astrofísica, Universidad de La Laguna, 38206, La Laguna, Tenerife, Spain

${ }^{4}$ Department of Science Education, Ewha Womans University, Seoul 120-750, Republic of Korea

${ }^{5}$ Department of Astronomy, University of Washington, Seattle, WA 98195, USA

${ }^{6}$ Astroparticule et Cosmologie (APC), Université Paris-Diderot, 75205 Paris Cedex 13, France

${ }^{7}$ CEA, Centre de Saclay, Irfu/SPP, F-91191 Gif-sur-Yvette, France

${ }^{8}$ Instituto de Física, UFRGS, Porto Alegre, RS 91501-970, Brazil

${ }^{9}$ Laboratório Interinstitucional de e-Astronomia (LIneA), Rio de Janeiro, RJ 20921-400, Brazil

${ }^{10}$ Department of Physics \& Astronomy and JINA: Joint Institute for Nuclear Astrophysics, Michigan State University, E. Lansing, MI 48824, USA

${ }^{11}$ Department of Physics and Astronomy, Vanderbilt University, Nashville, TN 37235, USA

${ }^{12}$ Department of Astrophysical Sciences, Princeton University, Princeton, NJ 08544, USA ${ }_{13}$ Apache Point Observatory, Sunspot, NM 88349, USA

${ }^{14}$ Center for Cosmology and Particle Physics, New York University, New York, NY 10003, USA

${ }^{15}$ Department of Astronomy and Astrophysics, 525 Davey Laboratory, The Pennsylvania State University, University Park, PA 16802, USA

${ }^{16}$ Department of Physics and Astronomy, University of Utah, Salt Lake City, UT 84112, USA

${ }^{17}$ Institute for Gravitation and the Cosmos, The Pennsylvania State University, University Park, PA 16802, USA

${ }^{18}$ Institute of Cosmology and Gravitation (ICG), Dennis Sciama Building, University of Portsmouth, Portsmouth, PO1 3FX, UK

${ }^{19}$ Department of Astronomy, University of Wisconsin-Madison, Madison, WI 53703, USA ${ }^{20}$ Astrophysical Institute Potsdam, 14482 Potsdam, Germany

${ }^{21}$ Istituto Nazionale di Astrofisica, 34143 Trieste, Italy

${ }^{22}$ Laboratoire d'Astrophysique de Marseille, CNRS-Université de Provence, 13388 Marseille Cedex 13, France

${ }^{23}$ Department of Physics, Hamilton College, Clinton, NY 13323, USA

${ }^{24}$ Lawrence Berkeley National Laboratory, Berkeley, CA 94720, USA

${ }^{25}$ Bruce and Astrid McWilliams Center for Cosmology, Carnegie Mellon University, Pittsburgh, PA 15213, USA 


\author{
${ }^{26}$ Yale Center for Astronomy and Astrophysics, Yale University, New Haven, CT 06520, USA \\ ${ }^{27}$ Observatório Nacional, Rio de Janeiro, RJ 20921-400, Brazil \\ ${ }^{28}$ Centre de Physique des Particules de Marseille, Aix-Marseille Université, CNRS/IN2P3, Marseille, France \\ ${ }^{29}$ Department of Astronomy, MSC 4500, New Mexico State University, Las Cruces, NM 88003, USA \\ ${ }^{30}$ Steward Observatory, Tucson, AZ 85721, USA \\ ${ }^{31}$ Harvard College Observatory, Cambridge, MA 02138, USA \\ ${ }^{32}$ Institut de Ciències de l'Espai (IEEC/CSIC), Campus UAB, E-08193 Bellaterra, Barcelona, Spain \\ ${ }^{33}$ Department of Physics \& Astronomy, Texas Christian University, Fort Worth, TX 76129, USA \\ ${ }^{34}$ Department of Astronomy, University of Florida, Bryant Space Science Center, Gainesville, FL 32611-2055, USA \\ ${ }^{35}$ Institute of Astronomy, University of Cambridge, Cambridge, CB3 OHA, UK \\ 36 Department of Astronomy, Ohio State University, Columbus, OH 43210, USA \\ ${ }^{37}$ Astronomisches Rechen-Institut, Zentrum für Astronomie der Universität Heidelberg, 69120 Heidelberg, Germany \\ ${ }^{38}$ Department of Astronomy, Case Western Reserve University, Cleveland, OH 44106, USA \\ ${ }^{39}$ Department of Astronomy, University of Virginia, Charlottesville, VA 22904-4325, USA \\ ${ }^{40}$ Department of Physics, Ohio State University, Columbus, OH 43210, USA \\ ${ }^{41}$ Research Center for the Early Universe, Graduate School of Science, The University of Tokyo, Bunkyo, Tokyo 113-0033, Japan \\ ${ }^{42}$ Department of Physics and Astronomy, University of California, Irvine, CA 92697, USA \\ ${ }^{43}$ Texas Advanced Computer Center, University of Texas, Austin, TX 78758-4497, USA \\ ${ }^{44}$ Observatories of the Carnegie Institution of Washington, Pasadena, CA 91101, USA \\ ${ }^{45}$ Fermi National Accelerator Laboratory, Batavia, IL 60510, USA \\ ${ }^{46}$ Department of Astronomy and Astrophysics, University of Chicago, Chicago, IL 60637, USA \\ ${ }^{47}$ Institute of Astronomy and Astrophysics, Academia Sinica, Taipei 10617, Taiwan \\ ${ }^{48}$ INAF, Osservatorio Astronomico di Padova, 35122 Padova, Italy \\ ${ }^{49}$ Center for Exoplanets and Habitable Worlds, 525 Davey Laboratory, Pennsylvania State University, University Park, PA 16802, USA \\ ${ }^{50}$ ICRA-Centro Brasileiro de Pesquisas Físicas, Urca, Rio de Janeiro, RJ 22290-180, Brazil \\ ${ }^{51}$ IPAC, MS 220-6, California Institute of Technology, Pasadena, CA 91125, USA \\ ${ }^{52}$ CITA, University of Toronto, University of Toronto, Toronto, ON M5S 3H8, Canada \\ ${ }^{53}$ Center for Astrophysical Sciences, Department of Physics and Astronomy, Johns Hopkins University, \\ Baltimore, MD 21218, USA \\ ${ }^{54}$ Institució Catalana de Recerca i Estudis Avançats, Barcelona, Spain \\ 55 Institut de Ciències del Cosmos, Universitat de Barcelona/IEEC, Barcelona 08028, Spain \\ ${ }^{56}$ SETI Institute/NASA Ames Research Center, Moffett Field, CA 94035, USA \\ ${ }^{57}$ US Naval Observatory, Flagstaff Station, Flagstaff, AZ 86001-8521, USA \\ ${ }^{58}$ Department of Astronomy, University of Illinois, Urbana, IL 61801, USA \\ ${ }^{59}$ Université Paris 6, Institut d'Astrophysique de Paris, UMR7095-CNRS, F-75014 Paris, France \\ ${ }^{60}$ Max-Planck-Institut für Extraterrestrische Physik, 85748 Garching, Germany \\ ${ }^{61}$ Center for Astrophysics and Space Astronomy, University of Colorado, 389 UCB, Boulder, CO 80309, USA \\ 62 Instituto de Astrofisica de Andalucia (CSIC), E-18008, Granada, Spain \\ ${ }^{63}$ Institut Utinam, Observatoire de Besançon, Université de Franche-Comté, BP1615, F-25010 Besançon Cedex, France \\ ${ }^{64}$ Department of Physics, Drexel University, Philadelphia, PA 19104, USA \\ ${ }^{65}$ Max-Planck-Institut für Astronomie, D-69117 Heidelberg, Germany \\ ${ }^{66}$ Observatório do Valongo, Universidade Federal do Rio de Janeiro, 20080-090 Rio de Janeiro, Brazil \\ ${ }^{67}$ UCO/Lick Observatory, University of California, Santa Cruz, Santa Cruz, CA 95064, USA \\ ${ }^{68}$ Bldg. 510 Brookhaven National Laboratory Upton, NY 11973, USA \\ ${ }^{69}$ Indian Institute of Astrophysics, II Block, Koramangala, Bangalore 560 034, India \\ ${ }^{70}$ Physics Department, University of California, Berkeley, CA 94720, USA \\ ${ }^{71}$ Max-Planck-Institut für Astrophysik, D-85748 Garching, Germany \\ Received 2011 June 28; published 2011 August 5
}

Section 3.5 of Aihara et al. (2011) described various sources of systematic error in the astrometry of the imaging data of the Sloan Digital Sky Survey (SDSS). In addition to these sources of error, there is an additional and more serious error, which introduces a large systematic shift in the astrometry over a large area around the north celestial pole. The region has irregular boundaries but in places extends as far south as declination $\delta \approx 41^{\circ}$. The sense of the shift is that the positions of all sources in the affected area are offset by roughly 250 mas in a northwest direction. We have updated the SDSS online documentation ${ }^{72}$ to reflect these errors, and to provide detailed quality information for each SDSS field.

In the Seventh Data Release of the SDSS (Abazajian et al. 2009), the astrometric calibration was performed with respect to the second data release of the United States Naval Observatory (USNO) CCD Astrograph Catalog (UCAC2; Zacharias et al. 2004), and a supplemental set of UCAC results in an internal USNO product known as "r14." The UCAC r14 data were used for declinations northward of approximately $40^{\circ}-50^{\circ}$ depending on right ascension. However, in the SDSS Eighth Data Release (DR8), we did not use the UCAC r14 catalog at high declination, but instead used the USNO-B catalog (Monet et al. 2003). The UCAC and USNO-B systems have a relative systematic offset of about 250 mas. The UCAC system is in much better agreement with the Tycho- 2 system (Høg et al. 2000) of the Hipparcos astrometric satellite.

We have performed a detailed comparison of the large-scale differences in astrometry between the SDSS DR8 and the UCAC catalogs. In the regions not covered by UCAC2 (starting northward of roughly $41^{\circ}$ declination), the DR8 astrometry is offset in the mean 240 mas to the north and 50 mas to the west relative to the r 14 catalog. On scales of about 0.25 , the rms scatter around this offset is about 80 mas in the declination direction and 94 mas in the right ascension direction. Some of that scatter is coherent on larger

\footnotetext{
72 http://www.sdss3.org
} 

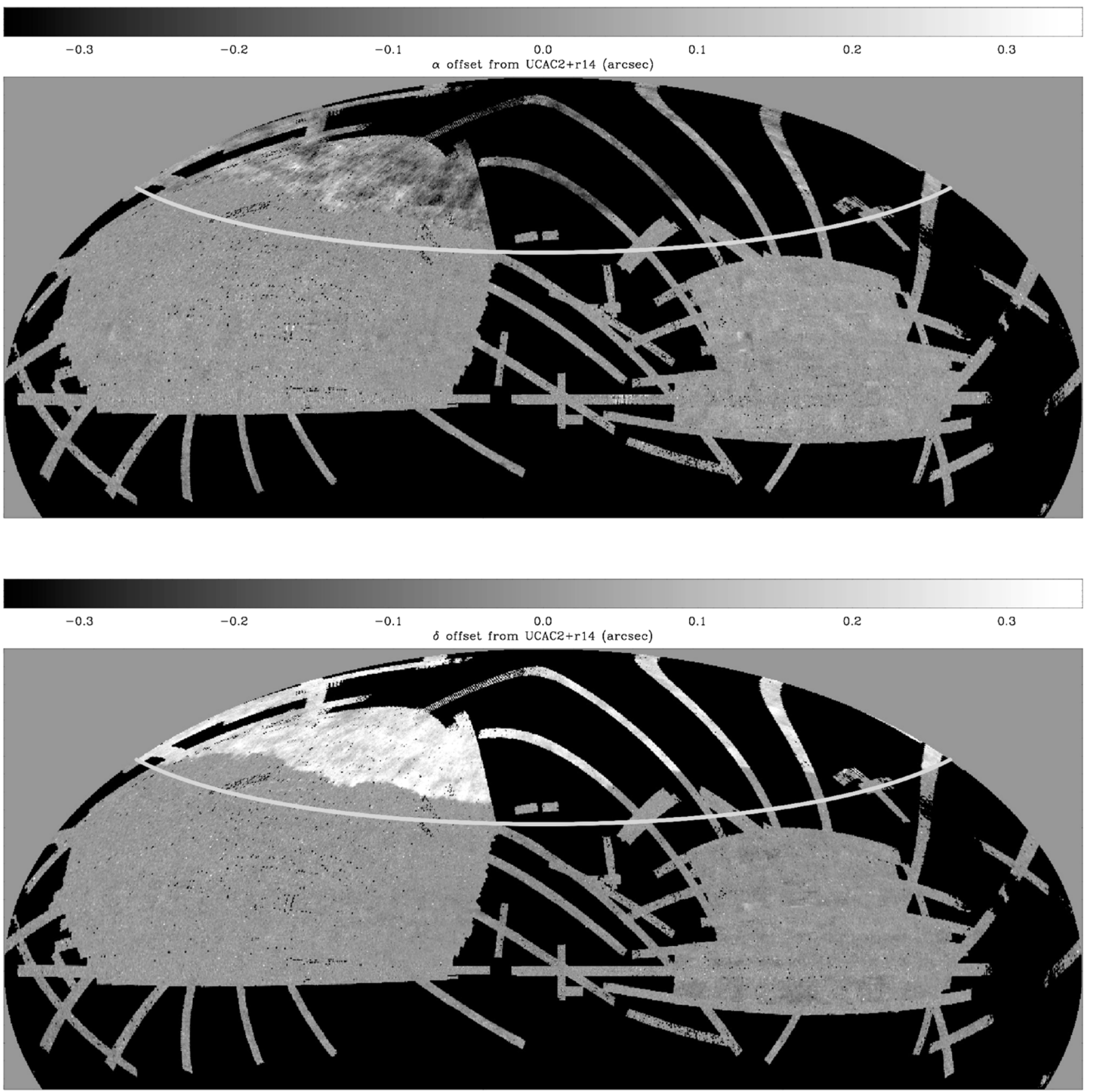

Figure 1. Difference between the coordinates of stars in the SDSS DR8 and those in UCAC2 (mostly south of $\delta=41^{\circ}$ ) and $\mathrm{r} 14\left(\right.$ mostly north of $\delta=41^{\circ}$ ), represented in gray scale as a function of right ascension and declination. The top panel shows differences in right ascension and the bottom panel shows differences in declination. The differences have been smoothed on scales of about 0.25 . The right ascension residuals are multiplied by $\cos \delta$ so that they are in units of proper angular distance. The residuals are shown in an Aitoff projection in equatorial coordinates. The gray line shows $\delta=41^{\circ}$. Black areas are outside the DR8 coverage.

scales; if we unsharp-mask by subtracting off the residual field smoothed with a Gaussian (FWHM $=3^{\circ}$ ), the remaining rms scatter is about 60 mas in either direction. A similar analysis south of $\delta=41^{\circ}$ yields very small offsets (less than 10 mas) between DR8 and UCAC2, with closer to the expected level of scatter (40 mas), and with no large-scale coherence to the scatter. These quantities include the effects of the systematic errors described in Section 3.5 of Aihara et al. (2011).

Figure 1 shows the nature and pattern of the DR8 offsets relative to the UCAC and r14 catalogs as a function of position on the sky.

The effect on the proper motions published in DR8 of the new errors described here is relatively small, because the proper motions in both DR7 and DR8 are calculated relative to USNO-B anyway (using local recalibrations). However, as noted in Section 3.5, the other errors in astrometry do have an effect on the proper motions. In the region with large astrometric errors in DR8, there is no

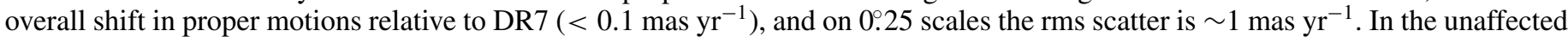

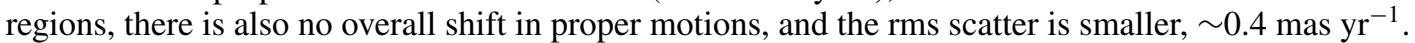


We recommend users requiring correct global astrometry in the affected areas to use DR7 astrometry where available; we provide matches to DR7 in the DR8 Catalog Archive Server (in the photoPrimaryDR7 and photo0bjDR7 tables). We are repairing the errors in the DR8 astrometry and will publish improved astrometric quantities and proper motions.

\section{REFERENCES}

Abazajian, K., Adelman-McCarthy, J. K., Agüeros, M. A., et al. 2009, ApJS, 182, 543

Aihara, H., Allende Prieto, C., An, D., et al. 2011, ApJS, 193, 29

Høg, E., Fabricius, C., Makarov, V. V., et al. 2000, A\&A, 355, L27

Monet, D. G., Levine, S. E., Canzian, B., et al. 2003, AJ, 125, 984

Zacharias, N., Urban, S. E., Zacharias, M. I., et al. 2004, AJ, 127, 3043 